\title{
Additions and corrections to the new Catalogue of Palaearctic Cerambycidae (Coleoptera) edited by I. Löbl and A. Smetana, 2010
}

\author{
Аополнения и исправления к новому каталогу палеарктических \\ Cerambycidae (Coleoptera) изданном I. Löbl и A. Smetana, 2010
}

\author{
M.L. Danilevsky

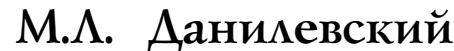

\begin{abstract}
A. N. Severtzov Institute of Ecology and Evolution, Russian Academy of Sciences, Leninsky prospect 33, Moscow 119071, Russia. E-mail: danilevskyml@rambler.ru,danilevsky@cerambycidae.net

Институт Проблем Экологии и Эволюции им. А.Н. Северцова РАН, Ленинский пр. 33, Москва 119071, Россия.
\end{abstract}

\begin{abstract}
KEY WORDS: Cerambycidae, taxonomy, new combinations, new synonyms, new names, Palearctic.
КЛЮЧЕВЫЕ СЛОВА: Cerambycidae, таксономия, новые сочетания, новые названия, Палеарктика.
\end{abstract}

ABSTRACT. For Volume 6 of Catalogue of Palaearctic beetles [2010] more than 150 misprints, wrong combinatiobns, wrong geographical records, wrong references, wrong status of certain names, wrong synonyms, wrong authotships and dates of certain names, wrong original combinations, wrong spelling of several names and so on are fixed. Sometimes unavailable names were publisjed as available. Missing names, geographical data and references are added. Natural system is proposed for several genera: Stenocorus, Acmaeops, Dinoptera, Carilia, Leptura, Cerambyx, Molorchus, Callimus, Phymatodes, Plagionotus, Xylotrechus, Saperda, Agapanthia. A replacement name is proposed for Leptura bisignata Brullé, 1832 (not Leptura bisignata Ménétriés, 1832): Vadonia grandicollis Mulsant \& Rey, 1863. The name Cortodera tibialis (Marseul, 1876) is proposed as valid for C. pallipipes Pic, 1898 (= C. ruthena Plavilstshikov, 1936). Pseudodinoptera Pic, 1900s is raised to genus level from a subgenus of Dinoptera Mulsant, 1863. Cortodera alpina xanthoptera Pic, 1898 is restored as a Turkish subspecies following Plavilstshikov [1936]. Alosterna tabacicolor tokatensis Pic, 1901 is accepted as a pale Turkish subspecies from Tokat and Erzincan. Dorcadion nigrosuturatum Reitter, 1897 is raised to species level from a subspecies of D. indutum Faldermann, 1837.

РЕЗЮМЕ. Для изданного в 2010 г. 6 тома каталога жуков Палеарктики приводятся исправления более 150 различных ошибок: опечаток, неправильных сочетаний, синонимов, ошибочных географических указаний, ошибочно указанных дат публикации названий и их авторов, ошибочных оригинальных сочетаний, написаний и т. д. Указаны несколько непригодных названий, опубликованных как пригодные. Добавлены пропущенные названия, публикации и географические данные. Естественные системы предложены для следующих родов: Stenocorus, Acmaeops, Dinoptera, Carilia, Leptura, Cerambyx, Molorchus, Callimus, Phymatodes, Plagionotus, Xylotrechus, Saperda, Agapanthia. Замещающее название предложено для Leptura bisignata Brullé, 1832 (не Leptura bisignata Ménétriés, 1832): Vadonia grandicollis
Mulsant \& Rey, 1863. Название Cortodera tibialis (Marseul, 1876) предложено в качестве валидного для C. pallipipes Pic, 1898 (=C. ruthena Plavilstshikov, 1936). Pseudodinoptera Pic, 1900s признан родом (ранее подрод в роде Dinoptera Mulsant, 1863). Cortodera alpina xanthoptera Pic, 1898 восстановлена в качестве турецкого подвида вслед за Плавильщиковым [1936]. Alosterna tabacicolor tokatensis Pic, 1901 принята как светло окрашенный турецкий подвид из Токата и Ерзинжана. Dorcadion nigrosuturatum Reitter, 1897 признан самостоятельным видом, а не подвидом D. indutum Faldermann, 1837.

The new Catalogue of Palaearctic Cerambycidae (Coleoptera) edited by I. Löbl and A. Smetana [2010] was prepared with the participation of 11 authors: K. Adlbauer, M.L. Danilevsky, A. Drumont, L. Hubweber, Z. Komiya, I. Löbl, J. Morati, P. Rapuzzi, G. Sama, A. Smetana and A. Weigel. The system of the authorship was rather complicated. It was partly arranged in taxonomy way and partly in geographical. So, such groups as Prioninae or Dorcadionini had own authors, while other authors were responsible for a certain geographical areas. The taxa of Western Palaearctic, for example, were arranged by G. Sama and I. Löbl, while M.L. Danilevsky and A. Smetana were responsible for the taxa from Russia and countries of the former USSR, and Mongolia. So, the authorship of all Cerambycidae genera, which are distributed in West Europe and in Russia was in fact mutual (with the exception of Dorcadionini). Each author had his own point of view on the system of each subfamily and each genus. So, the editors had to create a generally accepted system on the base of several different positions. As a result I. Löbl and A. Smetana took part in the arrangement of nearly each genus.

The necessity to join together several rather different texts (certain portions were presented to the editors in last moment before the publication) was the primary cause of many mistakes and misprints. Several names are published 
contemporary in different genera, the status of others was wrongly interpreted, many names were wrongly spelled or with wrong authors and dates, several geographical records were so strange, that could be simply misprints, many references were wrong, several publications were referred with two different names and so on. The main purpose of the present publication is to fix direct mistakes.

More over the system of such genera as Molorchus, Plagionotus, Xylotrechus, Saperda, Agapanthia and some others was accepted by editors on the base on the position by G. Sama, which was not natural and so not acceptable. The natural system of such groups is proposed bellow.

The references to the present article include only the publications absent in the references to the Catalog. The references inside the text of the present article to the publications included in the references to the Catalog have same letters after the number of the year as in the Catalog.

The style of a current paper in whole corresponds to the text of Catalogue of Palaearctic beetles [Vol.6: 2010]. All changes (additions, corrections and etc.) are underlined. Moreover, all of them are regularly published and updated in http://cerambycidae.net/.

\section{PAGES 11 AND 13}

The text of "DISTRIBUTIONAL INFORMATION" for Europe [P.11] in the present Vol. 6 is just same as in other volumes 1-5, but eastern boundaries of Europe in the map [P.13] are considerably changed. Before, the total territories of Ekaterinburg and Chelyabinsk regions (eastwards from Urals) were in Europe "CT", as well as the whole Orenburg region "ST". But now the eastern boundaries of Europe are marked along the main ridge of the Ural Mountains and along Ural river. So, now Ekaterinburg and Chelyabinsk regions are mostly (but not totally!) in Asia "WS", as well as the eastern part of Orenburg region (eastwards Ural River) "WS". The attribution of the south part of Orenburg region (southwards Ural River with several endemic taxa) is not clear in the Vol. 6 .

As far as I know most of authors (including me) were not informed on such modification. My distributional data in Vol. 6 were arranged in agreement with eastern boundaries of Europe shown in the maps for volumes 1-5: with Ekaterinburg and Chelyabinsk regions in Europe "CT", as well as the whole Orenburg region "ST".

\section{PAGE 42}

PRINTED:

Microarthron komarowi (Dohrn, 1885): the original spelling of the species epithet is komaroffi, a patronym in honour of General Komaroff. This spelling was also used by Heyden [1885b] but subsequent authors spelled the name "komarowi". The spelling "komarowi" is in prevailing use, and thus considered as correct [ICZN, Art. 33.3.1].

NOTE:

In fact, "komarowi" is not in prevailing usage! See: "komarovi" by Plavilstshikov [1932, 1936]; Kostin [1973]; Lobanov et al. [1981]; Mamaev, Danilevsky [1975]; Danilevsky [1984]; Śvácha [1987] — and many others!

So, it is better now to return to the original spelling: Microarthron komaroffi (Dohrn, 1885).

\section{PAGE 44} PRINTED:

Dorcadion sulcipenne argonauta Suvorov, 1913 as subspecies from species, based on the type examination of
D. argonauta Suvorov, 1913 (Zoological Institute, Sankt-Petersburg, Russia), and original description of $D$. sulcipenne Küster, 1847 as well as on numerous specimens of both taxa. NOTE:

Published before by Lazarev [2008].

\section{PAGE 44}

PRINTED:

Agapanthia subnigra Pic, 1890 is a valid species, as well as Agapanthia subchalydaea Reitter, 1898, though it was traditionally [Plavilstshikov, 1958a: 159] regarded as an invalid synonym of the junior name (based on the type material of both species).

NOTE:

Published before by Pesarini \& Sabbadini [2004b].

\section{PAGES $44-45$}

PRINTED:

Dorcadion sareptanum kubanicum Plavilstshikov, 1934: According to Plavilstshikov [1958a: 181] the male syntypes of Dorcadion euxinum Suvorov, 1915 (described from Novorossijsk) are $D$. sareptanum Kraatz, 1873, and only one female designated as type although not mentioned in the original description is $D$. cinerarium (Fabricius, 1787). Consenquently, Danilevsky et al. [2005] established the synonymy of D. sareptanum euxinum Suvorov and D. kubanicum Plavilstshikov, 1934. A study of the available syntypes of D. euxinum (housed in ZIN, Saint-Petersburg) revealed that they are all males of $D$. cinerarium. One of them is designated by Lazarev [in press] as lectotype. Thus, D. cinerarium (Fabricius) $=D$. euxinum Suvorov, and D. sareptanum $k u$ banicum Plavilstshikov is a valid name. The name $D$. euxinum was published as a synonym of $D$. cinerarium [Plavilstshikov, 1931: 64; 1958a: 118]

MUST BE:

Dorcadion sareptanum euxinum Suvorov, 1915: According to Plavilstshikov [1958a: 181] the male-syntypes of Dorcadion euxinum Suvorov, 1915 (described from Novorossijsk) are $D$. sareptanum Kraatz, 1873, and at least one type-female is $D$. cinerarium (Fabricius, 1787). Consequently, Danilevsky et al. [2005] established the synonymy of $D$. sareptanum euxinum Suvorov and D. kubanicum Plavilstshikov, 1934. In fact $D$. euxinum was described on the base of a single male, and that holotype was studied by Plavilstshikov [1958a], but recently (2009) was not found. Two available females (ZIN — designated as male and female) are wrongly designated by Suvorov as types of his D. euxinum, as both are not mentioned in the original description. The female designated by Suvorov as male is not the holotype, as it is much bigger $(14 \mathrm{~mm}$, while the holotype was $11.5 \mathrm{~mm})$ and has many different characters. The name $D$. euxinum was several times published by Plavilstshikov [1921: 111; 1931: 64; 1958a: 118] as a synonym of $D$. cinerarium, because Plavilstshikov accepted wrongly designated females as types of $D$. euxinum.

\section{PAGE 46}

PRINTED:

Dorcadion arietinum strandi Plavilstshikov, 1931, syn. nov. of Dorcadion arietinum phenax Jakovlev, 1900, based on examinations of respective type material and specimens from NW China.

NOTE:

The synonyms were published before by Breuning [1962a: 230] in form: "D. phenax m. strandi Plav." and then by Danilevsky [2009a:653; 2009b: 710] 


\section{PAGE 46}

PRINTED:

Leptura apicalis Motschulsky, 1875 syn. nov. of Stictoleptura fulva (DeGeer, 1775), based on examination of type materials of L. apicalis and West European Stictoleptura fulva. NOTE:

Published before by Lazarev [2008].

\section{PAGE 46}

PRINTED:

Macrorhabdium Plavilstshikov, 1915, syn.n. of Pseudosierversia Kraatz, 1879, based on study of the respective type species.

NOTE:

Macrorhabdium ruficolle Plavilstshikov, 1915, syn.n. of Pseudosierversia rufa Kraatz, 1879, based on the examination of the holotype of $P$. ruficolle and specimens of $P$. rufa from the Far East.

Published before by Danilevsky [2009a:633; 2009b: 692].

\section{PAGE 47}

PRINTED:

Pterolophia multinotata Pic, 1931 is senior synonym of Pterolophia mandshurica Breuning, 1938; it is used as valid, based on original description.

NOTE:

Published before by Lazarev [2008].

\section{PAGE 48}

PRINTED:

Nomen dubium Judolia tibialis Marseul, 1876 was described from Sarepta (Volgograd in Russia) but is currently placed in synonymy with Cortodera alpina alpina (Ménétriés, 1832) where the Caucasian C. alpina does not occur. Besides, the original description does not fit any Palaearctic Cerambycidae. The pronotal "angles postérieurs avancés en épine" exclude it from Cortodera Mulsant, 1863. However, it may have been based on an aberrant specimen Cortodera occuring in the area, eventually on a member of $C$. ruthena Plavilstshikov, 1936.

NOTE:

The name C. ruthena Plavilstshikov, 1936 is used here according to my old proposal [published by Danilevsky, 2009] to regard it as "nomen protectum", as well as Cortodera umbripepennis var. pallidipes Pic, $1898 \mathrm{~g}$ as "nomen oblitum" on the base of the Article 23.9 of ICZN [1999], though the necessary number of the articles by 25 authors for the last 50 years was not listed. The name Cortodera pallidipes Pic, 1898g is used in the Catalog [P.123] as valid without any special Act.

In fact the name Cortodera tibialis (Marseul, 1876) must be accepted as valid for the species. The poor level of the original description is not the reason to regard it as "nomen dubium".

\section{PAGE 51}

PRINTED:

Helladia iranica Villiers, 1960 and Helladia natali Lobanov, 1994, syn.n. of Helladia armeniaca testaceovittata Pic, 1934. The type specimen of Musaria testaceovittata, described from "Kojim, Lac Urmia" and currently regarded as species incertae sedis [Breuning, 1951], has recently been located in the Zoological Museum of Moscow University. It clearly belongs to Helladia Fairmaire, and agrees with the same species subsequently described as $H$. iranica and $H$. natali. Özdikmen [2008d] created the name Helladia armeniaca holzs- chuhi as a replacement name for $H$. armeniaca iranica Holzschuh. This is an obvious misunderstandig which cannot be explained, since Holzschuh neither described $H$. armeniaca iranica, nor mentioned such a name in the article quoted by Özdikmen. Helladia armeniaca holzschuhi Özdikmen, 2008 is to be consequently regarded as nomen nudum, since it was created as a replacement name for a non existing name. NOTE:

Females (a single female is known in P. testaceovittata natali) of $P$. t. testaceovittata and Ph. t. natali considerably differs by very wide prothorax, wider body and shorter antennae, besides the locality of $P h$. natali is strongly distant from the area (Iran) of Ph. testaceovittata. So, synonyms Ph. testaceovitata $($ Pic, 1934) $=$ Ph. natali Lobanov, 1994 can not be accepted. Ph testaceovitta natali is a northern subspecies.

\section{PAGE 52}

PRINTED:

Phytoecia (Blepisanis) vittipennis leuthneri (Ganglbauer, 1885)

MUST BE: 1886

Phytoecia (Blepisanis) vittipennis leuthneri Ganglbauer,

\section{PAGE 53}

PRINTED:

Sphenaria Pic, 1911, syn.n. of Pedostrangalia Sokolov, 1897 . The type species is $P$. revestita by monotypy which makes Sphenaria a synonym of Pedostrangalia. Furthermore, Sphenaria is a homonym of Sphenaria Mannerheim, 1849 (Coleoptera: Tenebrionidae).

NOTE:

It was not a synonym, but wrong subsequent spelling of Sphenalia (so unavailable). The name was not introduced as new: "La L. revestita L., rentrant dans le s.g. Sphenaria...".

\section{PAGE 54}

PRINTED:

Coptosia (Barbarina) chehirensis Breuning, 1943 MUST BE:

Coptosia (Barbarina) chehirensis (Breuning, 1943)

\section{PAGE 56}

PRINTED:

Phytoecia subannulipes Pic, 1910h: 51 from "Roumanie: Comana Vlasca" was never described. Pic [1910] mentioned $P$. subannulipes as described from "Syrie" and compared to it a female from "Roumanie: Comana Vlasca". In fact he compared P. subannulipes to itself: "Phytoecia subannulipes Pic. Cette espèce décrite de Syrie se retrouve en Roumanie ... l'a recueillie à Comana Vlasca. La femelle envoyée [.. ] ne diffère sensiblement des types, elle est seulement un peu plus petite et moins pubescente". Later on Pic [1911a: 9] wrote: "Phytoecia subannulipes Pic. Suivant la note de l'Echange $\mathrm{N}^{\circ} 307$ cette espèce syrienne se retrouverait en Roumanie" and again [Pic, 1915e: 11]: "P. subannulipes Pic, 1901, originaire de Syrie: on doit lui rapporter, comme varieté, subannulipes Pic, de Roumanie". Because of an evident lapsus and absence of a description, $P$. subannulipes is a nomen nudum.

NOTE:

The introduction (followed with morphological description) of the name "Phytoecia subannulipes" by Pic, $1910 \mathrm{~h}$ ("Cette espèce décrite de Syrie...") was undoubtedly a wrong spelling of Ph. subannularis Pic, $1901 \mathrm{~b}$ which was really "décrite de Syrie". It was repeated in form "Phytoecia subannulipes" once more [Pic, 1911a: 9]. But later Pic [1915f: 11] 
declared that Ph. subannulipes is a Roumanien variation of Ph. subannularis. So, the name became available in 1915 (as a synonym of $P h$. icterica).

According to G.Sama [personal communication, 2003], the records of the name for Roumania had to be connected with Ph. icterica (Schaller, 1783).

\section{PAGE 56}

PRINTED:

Leptura bisignata Brullé, 1832 and Leptura bisignata Ménétriés, 1832. Leptura bisignata Ménétriés takes priority over Leptura bisignata Brullé (currently in Vadonia Mulsant, 1863). However, the former name has never been regarded as valid after 1899 , being placed in synonymy with Stictoleptura tesserula (Charpentier, 1825). As both names apply to taxa considered congeneric after 1899 , the ICZN Art. 23.9.5. cannot be applied. The case should be referred to the Commission for a ruling. Meanwhile the name Leptura bisignata Brullé, currently in use, is maintained. NOTE:

The name Leptura bisignata Brullé, 1832 is a primary homonym [ICZN Art. 57.2]. It must be replaced if it is not published as valid in 25 publications by 10 authors for the last 50 years [ICZN Art. 23.9.1.2].

The replacement name is Vadonia grandicollis Mulsant \& Rey, 1863: 182 ("Les environs de Smyrne").

\section{PAGE 60}

PRINTED:

Dorcadion erytropteron Fischer von Waldheim, 1823 MUST BE:

Dorcadion erytropterum Fischer von Waldheim, 1823

\section{PAGE 85}

PRINTED:

genus Distenia Audinet-Serville, 1825: 485 type species Distenia columbina Audinet-Serville, 1825

Antinoe J. Thomson, 1864: 225 type species Antinoe bicolor J. Thomson, 1864

Apheles Blessig, 1872: 165 type species Apheles gracilis Blessig, 1872

Sakuntala Lameere, 1890: ccxiii type species Sakuntala kalidasae Lameere, 1890

Thelxiope J. Thomson, 1864: 226 type species Thelxiope viridicyanea J. Thomson, 1864

MUST BE:

genus Distenia Audinet-Serville, 1825: 485 type species Distenia columbina Audinet-Serville, 1825

Apheles Blessig, 1872: 165 type species Apheles gracilis Blessig, 1872

Basisvallis Santos-Silva \& Hovore, 2007:23 type species: Distenia agroides Bates, 1870

Sakuntala Lameere, 1890: ccxiii type species Sakuntala kalidasae Lameere, 1890

Thelxiope J. Thomson, 1864: 226 [HN] type species Thelxiope viridicyanea J. Thomson, 1864

Thomsonistenia Santos-Silva \& Hovore, 2007:3 [RN] type species: Thelxiope viridicyanea J. Thomson, 1864

subgenus Distenia Audinet-Serville, 1825: 485 type species Distenia columbina Audinet-Serville, 1825 NOTE:

Accordin to Santos-Silva \& Hovore [2007]: Antinoe J. Thomson, 1864 is a junior homonym, not Antinoe Kinberg, 1856 (Polychaeta: Polynoidae: Harmothoinae); new replacement name is Novantinoe Santos-Silva \& Hovore, 2007 - as another genus.
Accordin to Santos-Silva \& Hovore [2007]: Thelxiope J. Thomson, 1864 is a junior homonym, not Thelxiope Rafinesque-Schmaltz, 1814 (Crustacea).

Another subgenus was described:

Basisvallis Santos-Silva \& Hovore, 2007:23 type species:

Distenia agroides Bates, 1870.

The corresponding reference [Santos-Silva \& Hovore, 2007] was missing.

\section{PAGE 86}

PRINTED:

caspia Ménétriés, 1832: 225 E: AB A: IN

caspica Faldermann, 1835a: $261[\mathrm{HN}]$

MUST BE:

caspia Ménétriés, 1832: 225 E: AB A: IN

NOTE:

caspica Faldermann, 1837: 261 (wrong subsequent spelling) — not available.

\section{PAGE 87}

PRINTED:

sinicum validicornis Gressitt, 1951a: 205 (Megopis) A: JA

(Ishigaki-shima, Iriomote-shima)

MUST BE:

sinicum validicornis Gressitt, 1951ㅁ: 205 (Megopis) A: JA

(Ishigaki-shima, Iriomote-shima)

\section{PAGE 90}

PRINTED:

myardi myardi Mulsant, 1842a: 207 E: AL BH BU CR FR

GG GR IT PT SP TR UK YU N: AG EG LB MO TU A:

IN TR

abscisus Gilmour, 1954: 27 (Macrotoma)

gaubilii Chevrolat, 1859b: cxxxv

goudotii Chevrolat, $1859 \mathrm{c}$ : ccxxx

lethifer Fairmaire, 1859c: cxxxviii

scutellaris Germar, 1817: 219 [HN] (Prionus)

MUST BE:

myardi myardi Mulsant, 1842a: 207 E: AL BH BU CR FR GG

GR IT PT SP TR UK YU N: AG EG LB MO TU A: IN TR

abscisus Gilmour, 1954: 27 (Macrotoma)

gaubilii Chevrolat, 1859b: cxxxv

germari Mulsant, 1846: 291

goudotii Chevrolat, $1859 \mathrm{c}$ : ccxxx

lethifer Fairmaire, 1859c: cxxxviii

scutellaris Germar, 1817: 219 [HN] (Prionus)

\section{PAGE 90}

PRINTED:

pascoei pascoei Lansberge, 1884: 144 [RN] (Prinobius) A:

ANH AP BT FUJ GUA GUI GUX HAI HEB HP HUB

HUN NP SCH SD SHA UP XIZ YUN ZHE ORR

fisheri C. O. Waterhouse, 1884b: 382 (Macrotoma)

luzonum Pascoe, 1869: 666 [HN] (Macrotoma)

MUST BE:

pascoei pascoei Lansberge, 1884: 144 [RN] (Prinobius) A:

ANH AP BT FUJ GUA GUI GUX HAI HEB HP HUB

HUN NP SCH SD SHA UP XIZ YUN ZHE ORR

fisheri C. O. Waterhouse, 1884b: 382 (Macrotoma) NOTE:

Macrotoma luzonum, Pascoe, 1869: 666 was not a new name, but wrong identificaion!

\section{PAGE 92}

PRINTED:

zarudnii Semenov, 1933: 292 (Prionus) A: TD 
MUST BE:

zarudnii Semenov, 1933: 292 (Prionus) A: TD

zarudnyi Plavilstshikov, 1936: 80 (Prionus) [unjustified emendation]

\section{PAGE 92}

PRINTED:

komarowi Dohrn, 1885: 64 (Polyarthron) A: KZ TD TM UZ MUST BE:

komaroffi Dohrn, 1885: 64 (Polyarthron) A: KZ TD TM UZ komarovi Semenov, 1935b: 241, 246 (Prionus) [unjustified emendation]

komarowi Pic, 1898e: 33, 35 (Prionus) [unjustified emendation]

\section{PAGE 94}

PRINTED:

bienerti Heyden, 1885c: 311 (Polyarthron) A: IN TM banghaasi Pic, 1901i: 32 (Polyarthron) pluschewskyi Jakovlev, 1887a: 157 (Polyarthron)

MUST BE:

bienerti Heyden, 1885c: 311 (Polyarthron) A: IN TM banghaasi Pic, 1901i: 32 (Polyarthron) pluschewskyi Jakovlev, 1887a: 157 (Polyarthron) pluschtschewskii Semenov, 1900a: 252 (Polyarthron) [unjustified emendation]

plustschevskyi Semenov, 1935 (Prionus) [unjustified emendation]

pluschtschevskii Plavilstshikov, 1936 (Prionus) [unjustified emendation]

\section{PAGE 96}

PRINTED:

genus Alosterna Mulsant, 1863: 576 type species Leptura tabacicolor DeGeer, 1775

Allosterna Plavilstshikov, 1936: 302 [unjustified emendation]

MUST BE:

genus Alosterna Mulsant, 1863: 576 type species Leptura tabacicolor DeGeer, 1775

Allosterna Stierlin, 1898: 479 [unjustified emendation]

\section{PAGE 96}

PRINTED:

tabacicolor subvittata Reitter, 1885: 391 E: AB AR GG ST A: IN TR

caucasica Plavilstshikov, 1936: 305

tokatensis Pic, 1901n: 59

MUST BE:

tabacicolor subvittata Reitter, 1885: 391 E: AB AR GG ST

A: IN TR

caucasica Plavilstshikov, 1936: 305

tabacicolor tokatensis Pic, 1901n: 59 A: TR

NOTE:

Alosterna tabacicolor var. tokatensis Pic, 1901 (Turkey, Tokat) was described as a pale form with light $1^{\text {st }}$ antennal joint. I've got such specimens from near Erzincan - extreamly pale, not darkened along suture. A. t. tokatensis is not close to $A$. $t$. subvittata, neither to the nominative European subspecies.

\section{PAGE 100}

\section{PRINTED}

genus Gnathostrangalia Hayashi \& Villiers, 1985ㅁ: 13 type species Strangalia aurivillei Pic, 1903 MUST BE: genus Gnathostrangalia Hayashi \& Villiers, 1985ạ: 13 type species Strangalia aurivillei Pic, 1903

29. PAGE 101

PRINTED:

femorata Mulsant, 1863: 580 NOTE:

It was not a new name, but a wrong identification as Grammoptera femorata (Fabricius, 1787).

30. PAGE 101

PRINTED:

ruficornis obscuricornis Kraatz, 1886: 234 E: AB (Kavkaz) A: IN

ruficornis ruficornis Fabricius, 1781: 247 (Leptura) [NP] E:

AL AU BE BH BU BY CR CZ DE EN FR GB GE GR HU

IR IT LA LS LT MC MD NL NR PL PT RO SK SL SP ST SV SZ UK YU A: TR

MUST BE:

ruficornis obscuricornis Kraatz, 1886: 234 E: AB (Talysh) A: IN

ruficornis ruficornis Fabricius, 1781: 247 (Leptura) [NP] E:

AL AU BE BH BU BY CR CT CZ DE EN FR GB GE GR

HU IR IT LA LS LT MC MD NL NR PL PT RO SK SL SP ST SV SZ TR UK YU A: TR

NOTE:

The record for European Turkey see H. Özdikmen [2007], for Kaliningrad Region of Russia — Alekseev [2007].

31. PAGE 101

PRINTED:

ruficornis ruficornis Fabricius, 1781: 247 (Leptura) [NP] E:

AL AU BE BH BU BY CR CZ DE EN FR GB GE GR HU

IR IT LA LS LT MC MD NL NR PL PT RO SK SL SP ST

SV SZ UK YU A: TR

atra Fabricius, 1775: 197 (Leptura) [NO]

clavipes Geoffroy, 1785: 87 (Stenocorus)

laevis Herbst, 1784: 103 (Leptura)

pallipes Stephens, 1831: 264 (Leptura)

parisina Thunberg, 1784: 16 (Leptura)

pumila Schaller, 1783: 299 (Leptura)

rufipes Goeze, 1777: 501 (Leptura) [NO]

MUST BE:

ruficornis ruficornis Fabricius, 1781: 247 (Leptura) [NP] E:

AL AU BE BH BU BY CR CZ DE EN FR GB GE GR HU

IR IT LA LS LT MC MD NL NR PL PT RO SK SL SP ST

SV SZ UK YU A: TR

atra Fabricius, 1775: 197 (Leptura) [NO]

clavipes Geoffroy, 1785: 87 (Stenocorus)

holomelina Donisthorpe, 1905: 182

laevis Herbst, 1784: 103 (Leptura)

pallipes Stephens, 1831: 264 (Leptura)

parisina Thunberg, 1784: 16 (Leptura)

pumila Schaller, 1783: 299 (Leptura)

rufipes Goeze, 1777: 501 (Leptura) [NO] NOTE:

The name Grammoptera ruficornis ab. holomelina Pool, 1905 described from Great Britain is unavailable, though it was often used as valid. It was made available by Donisthorpe [1905] in same volum of same Journal, according to the Article 12.2 of ICZN, so such "indication" in the sense of that Article made Donisthorpe [1905] the author of the name. Totally black forms of G. ruficornis (with all legs also black) are not known from any other parts of the species area (neither in G. r. obscuricornis Kraatz, 1886 from Talysh and Iran). So, the problem with the validity of G. r. holomelina Donisthorpe, 1905 rests open. 


\section{PAGE 101}

PRINTED:

cyanea Tamanuki, 1933: 73 A: FE

MUST BE:

cyanea Tamanuki, 1933: 73 A: FE $\underline{\text { NE NC }}$

NOTE:

Grammoptera (Neoencyclops) cyanea was recorded for China by Hua [2002] as Grammoptera plavilstshikovi Heyrovsk1, 1965 and for North Korea by Tsherepanov [1996].

\section{PAGES 102 AND 116}

PRINTED:

$$
\text { p. } 102
$$

contracta Bates, 1884: 223 (Strangalia) A: JA JIX

ohbayashii Matsushita, 1933b: 220 (Strangalia)

tamanukii Hayashi, 1959b: 61 (Pygostrangalia) and p. 106

mediolineata Pic, 1954a: 13 A: JA

MUST BE:

contracta Bates, 1884: 223 (Strangalia) A: JA JIX

mediolineata Pic, 1954a: 13

ohbayashii Matsushita, 1933b: 220 (Strangalia)

tamanukii Hayashi, 1959b: 61 (Pygostrangalia) NOTE:

Idiostrangalia contracta $($ Bates, 1884) = Strangalia mediolineata Pic, 1954a [according to N. Ohbayashi, personal message, 2010].

34. PAGES 103, 105 AND 109-110

PRINTED: p. 103

genus Leptura Linnaeus, 1758: 397 type species Leptura quadrifasciata Linnaeus, 1758

Strangaliella Hayashi, 1976: 3 type species Strangalia shikokensis Matsushita, 1935 (= Strangalia tenuicornis Motschulsky, 1862 and p. 105

tenuicornis Motschulsky, 1862: 20 (Strangalia) A: JA shikokensis Matsushita, 1935: 309 (Strangalia) quadriluteonotata Pic, 1953a: 14 semisuturalis Pic, 1953a: 14

MUST BE:

$$
\text { p. } 109
$$

genus Parastrangalis Ganglbauer, 1889a: 57 type species Leptura potanini Ganglbauer, 1889

Strangaliella Hayashi, 1976: 3 type species Strangalia shikokensis Matsushita, 1935 (= Strangalia tenuicornis Motschulsky, 1862 and p. 110

sculptilis Holzschuh, 1991c: 30 A: SCH

shaowuensis Gressitt, 1951a: 112 (Strangalia) A: FUJ GUA HUB SCH

subapicalis Gressitt, 1935d: 263 (Strangalina) A: TAI

tenuicornis Motschulsky, 1862: 20 (Strangalia) A: JA quadriluteonotata Pic, 1953a: 14 (Leptura) semisuturalis Pic, 1953a: 14 (Leptura) shikokensis Matsushita, 1935: 309 (Strangalia)

35. PAGES 106 AND 107

PRINTED:

genus Macroleptura Nakane \& K. Ohbayashi, 1957: 241 type species Leptura thoracica Creutzer, 1799 quadrizona Fairmaire, 1902a: 244 (Strangalia) A: YUN ORR anticejuncta Pic, 1943c: 1 (Strangalia) magdelanei Pic, 1937b: 6 (Strangalia) thoracica Creutzer, 1799: 125 (Leptura) E: BH BY CT EN FI LA LT NT PL RO SK ST UK YU A: ES FE FUJ GUI HEB HEI HUB JA JIL KZ LIA MG NMO WS XIN ZHE "Korea"

altaica Gebler, 1817: 331 (Leptura)

obscurissima Pic, 1900i: 17 (Leptura)

maculiceps G. Schmidt, 1951: 12 (Strangalia)

mixtepilosa G. Schmidt, 1951: 12 (Strangalia)

ussurica Pic, 1902b: 8 (Leptura)

and

genus Noona Sama, 2007c: 102 [RN] type species Strangalia regalis Bates, 1884

Nona Sama, 2002: 25 [HN] type species Strangalia regalis Bates, 1884

regalis Bates, 1884: 223 (Strangalia) A: CH FE JA NC SC coreana Pic, 1907d: 20 (Leptura)

maindroni Pic, 1901m: 61 (Leptura)

MUST BE:

genus Leptura Linnaeus, 1758: 397 type species Leptura quadrifasciata Linnaeus, 1758

subgenus Macroleptura Nakane \& K. Ohbayashi, 1957: 241 type species Leptura thoracica Creutzer, 1799

quadrizona Fairmaire, 1902a: 244 (Strangalia) A: YUN ORR

anticejuncta Pic, 1943c: 1 (Strangalia)

magdelanei Pic, 1937b: 6 (Strangalia)

thoracica Creutzer, 1799: 125 (Leptura) E: BH BY CT EN FI LA LT NT PL RO SK ST UK YU A: ES FE FUJ GUI HEB HEI HUB JA JIL KZ LIA MG NMO WS XIN ZHE "Korea"

altaica Gebler, 1817: 331 (Leptura)

obscurissima Pic, 1900i: 17 (Leptura)

maculiceps G. Schmidt, 1951: 12 (Strangalia)

mixtepilosa G. Schmidt, 1951: 12 (Strangalia)

ussurica Pic, 1902b: 8 (Leptura)

and

subgenus Noona Sama, 2007c: 102 [RN] type species Strangalia regalis Bates, 1884

Nona Sama, 2002: 25 [HN] type species Strangalia regalis Bates, 1884

regalis Bates, 1884: 223 (Strangalia) A: CH FE JA NC SC coreana Pic, 1907d: 20 (Leptura) maindroni Pic, 1901m: 61 (Leptura)

36. PAGES 107

PRINTED:

genus Nivellia Mulsant, 1863: 564 type species Leptura sanguinosa Gyllenhal, 1827

subgenus Nivellia Mulsant, 1863: 564 type species Leptura sanguinosa Gyllenhal, 1827 and

subgenus Nivelliamorpha Boppe, 1921: 86 type species Leptura inequalithorax Pic, 1902 NOTE:

It is just a mistake. Genus Nivelliamorpha Boppe, 1921 has no connection with Nivellia Mulsant, 1863 becouse of wide and short body, totally different pronotal structure. It was published as a separtae genus long ago (Hayashi, 1987).

37. PAGES 110

PRINTED:

jaegeri Fairmaire, 1866b: 279 (Leptura)

MUST BE:

jaegeri Fairmaire, 1866b: 279 (Leptura) [HN] 


\section{PAGES 111 AND 837}

\section{PRINTED:}

orientalis Plavilstshikov, 1933a: 12 A: ES FE FUJ GAN HEB HEI HEN JA JIL LIA MG NC NMO SC SHA ZHE and

Plavilstshikov N.N. 1933a: Beitrag zur Verbreitung der paläarktischen Cerambyciden. III. Entomologisches Nachrichtenblatt 7: 9-16. — no new names here!

MUST BE:

orientalis Plavilstshikov, 1932: 114 A: ES FE FUJ GAN HEB HEI HEN JA JIL LIA MG NC NMO SC SHA ZHE NOTE:

The corresponding reference [Plavilstshikov, 1932] was missing.

\section{PAGES $113-114$}

PRINTED:

septempunctata septempunctata Fabricius, 1792b: 346 (Leptura) E: AL AU BH BU CR CZ GE GR HU IT MC MD PL RO SK SL ST SZ UK YU

atrosuturalis Pic, 1915a: 38 (Leptura)

corcyrica Pic, 1915e: 5 (Strangalia)

dobiachi Pic, 1916b: 4 (Strangalia)

gasturica Pic, 1915a: 38 (Leptura)

holtzi Pic, 1916b: 5 (Strangalia)

latenigra Pic, 1915e: 5 (Strangalia)

montandoni Pic, 1915e: 5 (Strangalia)

notaticollis Pic, 1915e: 5 (Strangalia)

pallidicolor Pic, 1915e: 5 (Strangalia)

roberti Pic, 1915a: 38 (Leptura)

rubronotata Pic, 1916b: 5 (Strangalia)

semireducta Pic, 1915e: 5 (Strangalia)

velebitica Pic, 1916b: 4 (Strangalia)

septempunctata suturata Reiche \& Saulcy, 1858: 22 (Strangalia) E: AR BU GG A: TR

anatolica Heyrovsk1, 1961a: 45 (Strangalia)

latenigra Pic, 1915e: 5 (Strangalia)

MUST BE:

septempunctata septempunctata Fabricius, 1792b: 346 (Leptura) E: AL AU BH BU CR CZ GE GR HU IT MC MD PL RO SK SL ST SZ UK YU atrosuturalis Pic, 1915a: 38 (Leptura) corcyrica Pic, 1915e: 5 (Strangalia) dobiachi Pic, 1916b: 4 (Strangalia) gasturica Pic, 1915a: 38 (Leptura) holtzi Pic, 1916b: 5 (Strangalia) montandoni Pic, 1915e: 5 (Strangalia) notaticollis Pic, 1915e: 5 (Strangalia) pallidicolor Pic, 1915e: 5 (Strangalia) roberti Pic, 1915a: 38 (Leptura) rubronotata Pic, 1916b: 5 (Strangalia) semireducta Pic, 1915e: 5 (Strangalia) velebitica Pic, 1916b: 4 (Strangalia)

septempunctata suturata Reiche \& Saulcy, 1858: 22 (Strangalia) E: AR BU GG A: TR

anatolica Heyrovsk1, 1961a: 45 (Strangalia)

latenigra Pic, 1915e: 5 (Strangalia)

\section{PAGE 115}

PRINTED:

fulva DeGeer, 1775: 137 (Leptura) E: AL AU BE BH BU BY CR CZ FR GB GE GR HU IR IT LS LU MC NL PT RO SK SL SP ST SZ TR UK YU A: TR

affinis Marsham, 1802: 353 (Leptura) apicalis Motschulsky, 1875: 142 (Leptura) corsica Pic, 1894k: 206 (Leptura) lutescens Geoffroy, 1785: 87 (Stenocorus) tomentosa Fabricius, 1792b: 340 (Leptura)

MUST BE:

fulva DeGeer, 1775: 137 (Leptura) E: AL AU BE BH BU BY

CR CZ FR GB GE GR HU IR IT LS LU MC NL PT RO

SK SL SP ST SZ TR UK YU A: TR

affinis Marsham, 1802: 353 (Leptura)

apicalis Motschulsky, 1875: 142 (Leptura)

corsica Pic, 1894k: 206 (Leptura)

fulvoapicalis Plavilstshikov, 1932: 174 (Leptura)

lutescens Geoffroy, 1785: 87 (Stenocorus)

tomentosa Fabricius, 1792b: 340 (Leptura) NOTE:

The corresponding references absent (see first note to the page 833): Plavilstshikov N.N. 1932: Lepturinen-Studien (Col., Cerambycidae). I. Časopis Československé Společnosti Entomologické 29: 87-88, 174-175.

\section{PAGE 115}

PRINTED:

maculicornis maculicornis DeGeer, 1775: 139 (Leptura) E:

AL AU BE BH BU BY CR CT CZ EN FI FR GE GG GR

HU IT LA LT LU MC MD NR NT PL RO SK SL ST SV SZ UK YU

MUST BE:

maculicornis DeGeer, 1775: 139 (Leptura) E: AL AU BE BH

BU BY CR CT CZ EN FI FR GE GG GR HU IT LA LT

LU MC MD NR NT PL RO SK SL ST SV SZ UK YU

42. PAGE 115

PRINTED:

rufa dimidiata K. Daniel \& J. Daniel, 1891: 11 (Leptura) A:

IN IQ TR

attaliensis K. Daniel \& J. Daniel, 1891: 11 (Leptura)

MUST BE:

rufa dimidiata K. Daniel \& J. Daniel, 1891: 11 (Leptura) A:

IN IQ TR

attaliensis K. Daniel \& J. Daniel, 1891: 11 (Leptura)

rubromarginata Plavilstshikov, 1932: 174 (Leptura) NOTE:

The corresponding references absent (see first note to the page 833): Plavilstshikov N.N. 1932: Lepturinen-Studien (Col., Cerambycidae). I. Časopis Československé Společnosti Entomologické 29: 87-88, 174-175.

\section{PAGE 117}

PRINTED:

bisignata bisignata Brullé, 1832: 264 (Leptura) E: BU GR grandicollis Mulsant \& Rey, 1863: 182

inapicalis Pic, 1897c: 31 (Leptura) [DA]

bisignata laurae Pesarini \& Sabbadini, 2007a: 25 E: GR

MUST BE (SEE NOTE 14 TO THE PAGE 56):

grandicollis grandicollis Mulsant \& Rey, 1863: 182 E: BU

GR A: TR

bisignata Brullé, 1832: 264 (Leptura) [HN]

inapicalis Pic, 1897c: 31 (Leptura) [DA]

grandicollis laurae Pesarini \& Sabbadini, 2007a: 25 E: GR NOTE:

Vadonia grandicollis Mulsant \& Rey, 1863 was described from «Smyrne» (Izmir).

44. PAGE 119

PRINTED:

genus Acmaeops LeConte, 1850a: 235 type species Leptura proteus Kirby, 1837

Gnathacmaeops Linsley \& Chemsak, 1972: 135 type species Leptura pratensis Laicharting, 1784 
MUST BE:

genus Acmaeops LeConte, 1850a: 235 type species Leptura proteus Kirby, 1837 and

genus Gnathacmaeops Linsley \& Chemsak, 1972: 135 type species Leptura pratensis Laicharting, 1784 NOTE:

See larval characters [Švácha, 1989: 87].

\section{PAGE 119}

PRINTED:

marginatus Fabricius, 1781: 247 (Leptura) E: AU BH BY CR CT CZ EN FI FR GE GR HU IT LA LT NL NR NT PL SK SL SP ST SV SZ UK YU A: ES FE GAN JA KZ MG NMO TR WS

MUST BE:

marginatus Fabricius, 1781: 247 (Leptura) E: AU BH BY CR CT CZ EN FI FR GE GR HU IT LA LT NL NR NT PL SK SL SP ST SV SZ UK YU A: ES FE GAN KZ MG NMO TR WS

\section{PAGE 119}

PRINTED:

angusticollis Gebler, 1833: 304 (Pachyta) E: CT NT PL A: ES FE JIL MG NC NMO SC WS XIN amurensis Suvorov, 1915: 346

sachalinensis Tsherepanov, 1978a: 99

MUST BE:

angusticollis Gebler, 1833: 304 (Pachyta) E: BY CT NT PL

A: ES FE JIL JP MG NC NMO SC WS XIN

amurensis Suvorov, 1915: 346

sachalinensis Tsherepanov, 1978a: 99

NOTE:

The record for Belorussia was published by Alexandrovich et al. [1996], for Japan (Hokkaido) — by M. Hayashi [1983] on the base of a single specimen.

The synonyms Pachyta angusticollis Gebler, $1833=$ Acmaeops sachalinensis Tsherepanov, 1978 were never published before, but corresponding comments absent in the Catalog.

I've studied (2001) the holotype male of Acmaeops sachalinensis (preserved in Zoological Institute in St.-Petersburg) with the label in Russian: "[Sakhalin, Nikolskiy Bay, Nikolsky leg.]" and another small lable with date: "17.4.09". It is a colourless specimen of $A$. angusticollis, so A. angusticollis $=A$. sachalinensis. There is also a series of similar colourles specimens of Gnathacmaeops pratensis with similar labels in Russian "[Sakhalin, Nikolsky leg.]" in the Museum.

\section{PAGE 119}

PRINTED

pratensis Laicharting, 1784: 172 (Leptura) E: AB AL AN AR AU BH BU BY CR CT CZ EN FI FR GE GG HU IT LA LT MC MD NR NT PL RO SK SL SP ST SV SZ YU UK A: ES FE KI KZ MG NMO SC UZ WS XIN NAR fulvipennis Mannerheim, 1853: 251 (Pachyta)

lateralis Estlund, 1796: 127 (Leptura)

longiceps Kirby, 1837: 187 (Leptura) semimarginatus Randall, 1838: 30 (Leptura) suturalis Mulsant, 1839: 246 (Pachyta) strigilatus Fabricius, 1792b: 341 (Leptura)

MUST BE:

pratensis Laicharting, 1784: 172 (Leptura) E: AB AL AN AR AU BH BU BY CR CT CZ EN FI FR GE GG HU IT LA LT MC MD NR NT PL RO SK SL SP ST SV SZ
YU UK A: ES FE KI KZ MG NMO SC UZ WS XIN NAR

lateralis Estlund, 1796: 127 (Leptura)

obscuripennis Pic, 1901: 24

suturalis Mulsant, 1839: 246 (Pachyta)

strigilatus Fabricius, 1792b: 341 (Leptura)

ustulatus Motschulsky, 1860: 148 (Pachyta)

NOTE:

three names belong to American species:

Acmaeops longiceps [Kirby, 1837: 187 - in Leptura].

fulvipennis Mannerheim, 1853: 251 (Pachyta)

semimarginatus Randall, 1838: 30 (Leptura)

two names were missing

obscuripennis Pic, 1901: 24

ustulatus Motschulsky, 1860: 148 (Pachyta)

48. PAGES 119, 134

PRINTED:

genus Anisorus Mulsant, 1862: 467 type species Cerambyx quercus Götz, 1783

MUST BE:

genus Stenocorus Geoffroy, 1762: 221 type species Leptura meridiana Linnaeus, 1758

subgenus Anisorus Mulsant, 1862: 467 type species Cerambyx quercus Götz, 1783

49. PAGE 120

PRINTED:

bifasciata bifasciata Olivier, 1792a: 520 (Leptura) A: ES FE GAN HEB HEI JIL LIA NMO QIN SC SCH XIZ

MUST BE:

bifasciata bifasciata Olivier, 1792a: 520 (Leptura) A: ES FE GAN HEB HEI JIL LIA MG NMO QIN SC SCH XIZ

50. PAGE 120

PRINTED:

bifasciata japonica Matsushita, 1933a: 178 (Evodinus) A: FE JA NMO

MUST BE:

bifasciata japonica Matsushita, 1933ㅁ: 178 (Evodinus) A: FE JA

\section{PAGE 120}

PRINTED:

caucasica caucasica Rost, 1892: 309 E: GG

MUST BE:

caucasica caucasica Rost, 1892a: 309 [1892b: 81] E: GG conjuncta Rost, 1893: 344

NOTE:

The corresponding reference [Rost C., 1892] was missing.

\section{PAGE 120}

PRINTED:

duodecimmaculata Fabricius, 1781: 248 (Leptura) [NO] MUST BE:

duodecimmaculata Fabricius, 1781: 248 (Leptura) NOTE:

Because the name is younger, than valid one - Brachyta interrogationis (Linnaeus, 1758). In fact the true origin of Leptura duodecimmaculata Fabricius, 1781: 248 is not clear.

53. PAGE 120

PRINTED:

kraatzi Ganglbauer, 1889c: 468 [RN]

MUST BE:

kraatzi Ganglbauer, 1889c: 468 
NOTE:

The name Brachyta punctata var. kraatzi Ganglbauer, 1889 is not a replacement name! It was proposed for the specimens from Amur river valley, which were wrongly identified (and described) by Solsky [1871: 397] as Pachyta interrogationis var. duodecimmaculata (Fabricius, 1781), while Solsky's identification of Mongolian specimens as $P$. $i$. var. duodecimmaculata (Fabricius, 1781) could be correct.

\section{PAGES 120 AND 121}

PRINTED:

bernardinus Pic, 1915a: 41 (Evodinus)

theresae Pic, 1915a: 41 (Evodinus)

NOTE:

Both names are unavailable as proposed for one population ("Alpes: Petit Saint-Bernard")

\section{PAGES 120 AND 121}

PRINTED:

immaculatus Pic, 1933i: 28

mulsanti Pic, 1933i: 31

multiguttatus Pic, 1933i: 31

plavilstshikovi Pic, 1933i: 31

prescutellaris Pic, 1933i: 31

MUST BE (SEE NOTE 106 TO THE PAGE 833):

immaculatus Pic, 1934f: 28 (Evodinus)

mulsanti Pic, 1934f: 31 (Evodinus)

multiguttatus Pic, 1934f: 31 (Evodinus)

plavilstshikovi Pic, 1934f: 31 (Evodinus)

prescutellaris Pic, 1934 f̂: 31 (Evodinus)

56. PAGE 121

PRINTED:

genus Brachyta Fairmaire, 1864a: 185 type species Leptura interrogationis Linnaeus, 1758

striolata Gebler, 1817: 330 (Leptura) A: ES MG

brevelineata Pic, 1926d: 10

eurinensis Tsherepanov, 1978a: 97 (Evodinus)

mutabilis Motschulsky, 1859a: 233 (Evodinus)

striatiformis Plavilstshikov, 1936: 196 (Evodinus)

variabilis phlaesa Z. Wang, 2003: 127, 398 (Evodinus) A: HEI

variabilis scapularis Mannerheim, 1849: 245 (Pachyta) A:

ES FE MG NE NMO

comosa Solsky, 1871a: 400 (Pachyta)

discobilineata Pic, 1928c: 2

heyrovskyi Pic, 1926d: 10

instriolata Pic, 1912c: 2 (Evodinus)

intermedia Pic, 1916b: 3 (Evodinus)

multisignata Pic, 1915a: 41 (Evodinus)

mutabilis Motschulsky, 1859a: 571 (Pachyta)

MUST BE:

genus Brachyta Fairmaire, 1864a: 185 type species Leptura interrogationis Linnaeus, 1758$$
\text { ... }
$$

striolata Gebler, 1817: 330 (Leptura) A: ES MG brevelineata Pic, 1926d: 10

eurinensis Tsherepanov, 1978a: 97 (Evodinus) striatiformis Plavilstshikov, 1936: 196 (Evodinus)

variabilis phlaesa Z. Wang, 2003: 127, 398 (Evodinus) A: HEI variabilis scapularis Mannerheim, 1849: 245 (Pachyta) A: ES FE MG NE NMO

comosa Solsky, 1871a: 400 (Pachyta)

discobilineata Pic, 1928c: 2

heyrovskyi Pic, 1926d: 10

instriolata Pic, 1912c: 2 (Evodinus)

intermedia Pic, 1916b: 3 (Evodinus)

multisignata Pic, 1915a: 41 (Evodinus)

mutabilis Motschulsky, 1859a: 571 (Pachyta) [1859a: 233 (Pachyta)

57. PAGE 121

PRINTED:

beckeri Desbrochers des Loges, 1875a: 51

MUST BE:

beckeri Desbrochers des Loges, 1875a: $51 \underline{\text { (Pachyta) }}$

58. PAGE 122

PRINTED:

confusa Reitter, 1891b: 34 (Cartodera)

MUST BE:

confusa Reitter, 1891b: 34

NOTE:

As «Cartodera Reitter, 1891b: 34» is unavailable wrong subsequent spelling.

59. PAGE 122

PRINTED:

alpina rosti Pic, 1892q: 1xxxiii E: ST (Kavkaz)

alpina starcki Reitter, 1888b: 280 E: GG ST (Kavkaz)

parallela Pic, 1898k: 111

MUST BE:

alpina rosti Pic, 1892q: 1xxxiii E: ST (Kavkaz)

parallela Pic, $1898 \mathrm{k}: 111$

alpina starcki Reitter, 1888b: 280 E: GG ST (Kavkaz) NOTE:

Cortodera ?starcki var. parallela Pic, 1898k: 111 ("Caucase") was described by Pic on the base of a single female with yellow elytra. All known $C$. alpina starcki are totally black. The female of var. parallela Pic was most probably collected in the North Caucasus and can be regarded as C. alpina rosti Pic, 1892q.

60. PAGE 122

PRINTED:

alpina umbripennis Reitter, 1890e: 245 E: AB AR GG ST A:

IN

armeniaca Pic, 1898k: 114

flavipennis Ganglbauer, 1897a: 53

rosinae Pic, 1902c: 8

xanthoptera Pic, 1898k: 115

MUST BE:

alpina umbripennis Reitter, 1890e: 245 E: AB AR GG ST A:

IN

armeniaca Pic, $1898 \mathrm{k}: 114$

alpina xanthoptera Pic, 1898k: 114 [RN]

flavipennis Ganglbauer, 1897a: 53 [HN] (not Cortodera

femorata var. flavipennis Reitter, 1890e: 243)

rosinae Pic, $1902 \mathrm{c}: 8$

NOTE:

C. alpina umbripennis is known from the regions of Turkey close to Transcaucasia. According to Plavilstshikov [1936: 289] Cortodera umbripennis ssp. xanthoptera Pic, 1898 is a taxon distributed in Anatolia and Syria. So, the valid name of the taxon is C. alpina xanthoptera Pic, 1898 


\section{PAGE 122}

PRINTED:

flavimana Waltl, 1838: 471 (Leptura) E: AU BU GR HU MC RO SK TR YU A: TR

brachialis Ganglbauer, 1897a: 52

flavipennis Ganglbauer, 1897a: 53

MUST BE:

flavimana Waltl, 1838: 471 (Leptura) E: AU BU GR HU MC RO SK TR YU A: TR

brachialis Ganglbauer, 1897a: 52

NOTE:

Same name as in the previous note 62 ! It was introduced as Cortodera flavimana var. flavipennis Ganglbauer, 1897a: 53. The taxon was moved to $C$. umbripennis with a replacement name: Cortodera umbripennis var. xanthoptera Pic, 1898: $114,115.117$. And then generally accepted in that position, see Aurivillius [1912], Winkler [1929], Plavilstshikov [1936].

\section{PAGE 122}

PRINTED:

analis Gebler, 1830: 189 (Pachyta) A: KZ WS XIN

haemorrhoidalis Pic, 1898k: 77

hirta Gebler, 1830: 190 (Leptura)

holosericea Gebler, 1848a: 423 (Leptura)

ruficornis Pic, 1926d: 6

MUST BE:

analis Gebler, 1830: 189 (Pachyta) A: KZ WS XIN

haemorhoidalis Pic, 1898k: 77

haemorrhoidalis Aurivillius, 1912: 197 [unjustified emendation]

hirta Gebler, 1830: 190 (Pachyta)

ruficornis Pic, 1926d: 6 NOTE:

First of all the name "holosericea" was published by Gebler in genus Grammoptera. It was not used as a new name but as " $G$. holosericea F." - wrong identification of his Pachyta analis.

\section{PAGE 122}

\section{PRINTED:}

colchica colchica Reitter, 1890e: 246 E: AB AR GG ST A:

IN LE SY TR

deyrollei Pic, 1894c: 66

distincta Pic, 1933d: 6

lederi Pic, 1933d: 6

ordubadensis Reitter, 1890e: 246

pseudalpina Plavilstshikov, 1936: 278

pygidialis Reitter, 1890e: 246

rutilipes Reitter, 1890e: 246

truncatipennis Pic, 1929h: 119 [DA]

MUST BE:

colchica colchica Reitter, 1890e: 246 E: AB AR GG ST A:

IN LE SY TR

atropyga Pic, 1929h: 119 [DA]

deyrollei Pic, 1894c: 66

distincta Pic, 1933d: 6

lederi Pic, 1933d: 6

ordubadensis Reitter, 1890e: 246

pseudalpina Plavilstshikov, 1936: 278

pygidialis Reitter, 1890e: 246

rutilipes Reitter, 1890e: 246

truncatipennis Pic, 1929h: 119 [DA]

64. PAGE 122

PRINTED:

colchica danczenkoi Danilevsky, 1985: 139 E: AB
MUST BE:

colchica danczenkoi Danilevsky, 1985: 139 [1987: 615] E: AB NOTE:

New descriptions [Danilevsky, 1987] were accepted for publication by "Revue d'Entomologie de l'URSS" 4 years before the publication. The same new taxa were included in a subsequent paper [Danilevsky \& Miroshnikov, 1985], providing keys. Consequently, the latter publication has priority, although lacking complete descriptions, illustrations and data on type materials.

\section{PAGE 122}

PRINTED:

griseipes Pic, 1889a: 55 (Grammoptera)

MUST BE:

griseipes Pic, 1889ㅁ: 55 (Grammoptera)

\section{PAGE 122}

PRINTED:

holosericea Fabricius, 1801b: 366 (Leptura) E: AU BH BU

HU CR GR HU IT RO SK SL ST UK YU

birnbacheri Pic, 1898k: 114

rubripes Pic, 1898k: 114

velutina Heyden, 1876a: 318

MUST BE:

holosericea holosericea Fabricius, 1801b: 366 (Leptura) E:

AU BU HU RO SK ST UK

pilosa Pic, 1989g: 50

rubripes Pic, 1898k: 114

semitestacea Pic, 1989g: 50

holosericea velutina Heyden, 1876a: 318 E: AU BH CR GR IT SL YU

birnbacheri Pic, 1898k: 114

NOTE:

See [Mikšić, 1971; Sama, 1988; Althoff \& Danilevsky, 1997; Illić, 2005]. Bisides, several other names could be valid.

\section{PAGES 122-123}

PRINTED:

discolor Fairmaire, 1866b: 277 A: TR

differens Pic, 1898g: 50

prescutellaris Pic, $1933 \mathrm{~d}: 5$

testaceipes Pic, 1898k: 112

and

steineri Sama, 1997b: 112 E: GR

MUST BE:

differens Pic, 1898g: 50 E: GR prescutellaris Pic, $1933 \mathrm{~d}$ : 5 steineri Sama, 1997b: 112 and

discolor Fairmaire, 1866b: 277 E: BG A: TR testaceipes Pic, 1898k: 112

\section{PAGE 123}

PRINTED:

humeralis orientalis Adlbauer, 1988: 264 A: TR

MUST BE:

orientalis Adlbauer, 1988: 264 A: TR NOTE:

According to justified opinion by Sama [2002: 21]: “Cortodera orientalis Adlbauer, 1988, described as a subspecies of $C$. humeralis, is a distinct species".

69. PAGE 123

PRINTED:

pallidipes komarovi Danilevsky, 1996c: 63 A: KZ 
pallidipes pallidipes Pic, 1898g: 49 E: ST A: KZ ruthena Plavilstshikov, 1936: 286 pallidipes rossica Danilevsky, 2001b: 7 E: UK ST pallidipes turgaica Danilevsky, 2001b: 9 E: CT A: KZ MUST BE:

tibialis komarovi Danilevsky, 1996c: 63 A: KZ tibialis ruthena Plavilstshikov, 1936: 286 E: ST A: KZ tibialis rossica Danilevsky, 2001b: 7 E: UK ST tibialis tibialis Marseul, 1876: cii (Judolia) E: ST pallidipes Pic, $1898 \mathrm{~g}$ : 49

tibialis turgaica Danilevsky, 2001b: 9 E: CT A: KZ NOTE:

See note to the page 48 for the validity of Cortodera tibialis (Marseul, 1876). The presence of totally black males and males with all legs black in Volgograd population - C. tibialis tibialis Marseul, 1876 shows its separate subspecies status. Such males art not known in the population from near Uralsk, neither from Orenburg - C. tibialis ruthena Plavilstshikov, 1936. A lot of females are collected now each year in Orenburg Region by different collectors, but no males are observed.

\section{PAGE 123}

PRINTED:

pseudomophlus Reitter, 1889a: 40 E: AR AB A: IN TM MUST BE:

pseudomophlus Reitter, 1889a: 40 E: AR AB A: IN TM TR NOTE:

The species was several times recorded for Turkey [Villiers, 1967: 348 — "Arménie turque"; Adlbauer, 1992: 490 - "Yenicekale W Kahramanmaras", "Askale, W Erzurum"; Özdikmen, 2003: 437].

\section{PAGE 123}

\section{PRINTED:}

schurmanni Sama, 1997b: 107 E: GR diversipes Pic, 1898k: 79

MUST BE:

diversipes Pic, 1898k: 79 E: GR

schurmanni Sama, 1997b: 107

\section{PAGE 123}

PRINTED:

parfentjevi Miroshnikov, 2007: 217 E: UK (Krym)

MUST BE:

parfentjevi Miroshnikov, 2007: $21 \underline{5}$ E: UK (Krym)

\section{PAGE P. 124}

PRINTED:

persica Plavilstshikov, 1936: 539

MUST BE:

persica Plavilstshikov, 1936: 291

\section{PAGE 124}

PRINTED:

subgenus Pseudodinoptera Pic, 1900s: 82 type species $A c$ maeops daghestanicus Pic, 1897

\section{MUST BE:}

Genus Pseudodinoptera Pic, 1900s: 82 type species Acmaeops daghestanicus Pic, 1897 NOTE:

Pseudodinoptera Pic, 1900 was described as a subgenus of Acmaeops, but soon [Pic, 1901: 23] was raised to genus level. That new status was not accepted by subsequent authors [Aurivillius, 1812; Winkler, 1929; Plavilstshikov, 1936], who continued to regard it as a subgenus of Acmaeops. The relocation of the subgenus to genus Dinoptera by Lobanov et al. [1981] can not be regarded as successful. Anyway Pseudodinoptera differs from Dinoptera by positions of antennal insertions similar to Gnathacmaeops, but has elongated body not tapering posteriorly, so it must be regarded as a genus.

The type series (from Shakhbuz Dag, Daghestan, Russia) of Acmaeops daghestanica Pic, 1897 (male and female) is preserved in the collection of Museum National d'Histoire Naturelle (Paris). The specimens are equipped with red labels:

male - "LECTOTYPE Pseudodinoptera daghestanica Pic G. SAMA DES 2004"

female - "PARALECTOTYPE Pseudodinoptera daghestanica Pic G. SAMA DES 2004"

Lectotype designation was not published.

\section{PAGE 124}

PRINTED:

sylvestris Geoffroy, 1785: 88 (Leptura)

MUST BE:

sylvestris Geoffroy, 1785: 88 ( $\underline{\text { Stenocorus })}$

\section{PAGE 124}

PRINTED:

frivaldskyi Kraatz, 1876ㅁ: 344 (Grammoptera)

MUST BE:

frivaldszkyi Kraatz, 1876: 318 (Grammoptera) NOTE:

The corresponding reference [Kraatz, 1876] was missing.

\section{PAGE 125}

PRINTED:

interruptelunata G. Schmidt, 1951: 6

MUST BE:

interruptelunata G. Schmidt, 1951: 11

\section{PAGE 125}

PRINTED:

borealis Gyllenhal, 1827: 36 (Leptura) E: BY CT EN FI LA LT NR NT PL SK SV UK A: ES FE JA MG NC NE NMO SC WS

brunneonotatus Pic, 1901b: 11 (Brachyta)

grisescens Pic, 1889b: 78 (Pidonia)

interruptelunata $\mathrm{G}$. Schmidt, 1951: 6

lateobscurus Pic, 1901b: 11 (Brachyta)

obscurissimus Pic, 1904a: 3

pallescens Fujimura, 1956: 2

pictus Mäklin, 1845: 549 (Pachyta)

MUST BE:

borealis Gyllenhal, 1827: 36 (Leptura) E: BY CT EN FI LA LT NR NT PL SK SV UK A: ES FE JA MG NC NE NMO SC WS

brunneonotatus Pic, 1901b: 11 (Brachyta)

grisescens Pic, 1889b: 78 (Pidonia)

interruptelunata G. Schmidt, 1951: 11

lateobscurus Pic, 1901b: 11 (Brachyta)

obscurissimus Pic, 1904a: 3

pallescens Fujimura, 1956: 2

pictus Mäklin, 1845: 549 (Pachyta)

schrammi Pic, 1945b: 6

separatus Pic, 1945b: 6

\section{PAGE 125}

PRINTED:

clathratus Fabricius, 1792b: 306 (Rhagium) E: AU BH BU CR CZ FR GE HU IT LS MC MD PL RO SK SL SZ UK YU 
atromultiplicatus Pic, 1945b: 5 atroreductus Pic, 1915a: 41 brunnipes Mulsant, 1839: 238 diversesignatus Pic, 1945b: 5 ...

MUST BE:

clathratus Fabricius, 1792b: 306 (Rhagium) E: AU BH BU CR CZ FR GE HU IT LS MC MD PL RO SK SL SZ UK YU atromultiplicatus Pic, 1945b: 5 atroreductus Pic, 1915a: 41 brunnipes Mulsant, 1839: 238 diversesignatus Pic, 1945b: 5 flecki G. Schmidt, 1958: 77

\section{PAGE 125}

PRINTED: nigritus Pic, 1891b: 6

MUST BE: nigritus Pic, 1891b: 6 (Brachyta)

81. PAGE 125

PRINTED:

elegans Faldermann, 1837: 319 (Grammoptera) E: AB AR CT GG ST A: IN TR

MUST BE:

elegans Faldermann, 1837: 319 (Grammoptera) E: AB AR GG ST A: IN TR NOTE:

No records of Fallacia elegans from Central Russia exists. The species does not occur northward Caucasian Region.

\section{PAGES 125-126}

PRINTED:

genus Gaurotes LeConte, 1850b: 324 type species Rhagium cyanipenne Say, 1824

subgenus Carilia Mulsant, 1863: 489 type species Leptura virginea Linnaeus, 1758

and

subgenus Gaurotes LeConte, 1850b: 324 type species

Rhagium cyanipenne Say, 1824 and

subgenus Paragaurotes Plavilstshikov, 1921: 116 type species Gaurotes ussuriensis Blessig, 1873 NOTE:

The genus Gaurotes is purely Nearctic [see Villiers, 1978: 123]. Paragaurotes and Carilia are separate genera.

\section{PAGE 126}

PRINTED:

virginea aemula Mannerheim, 1852b: 306 E: CT ST A: ES

FE HEI HUB JIL KZ MG NMO SHX WS

sibirica Podan1, 1962: 236 (Gaurotes)

virginea kozhevnikovi Plavilstshikov, 1915c: 105 (Gaurotes)

\section{A: FE HEI JIL NC SC}

komensis Tamanuki, 1938b: 167 (Gaurotes)

nigriventris Jureček, 1921: 25 (Gaurotes)

nigriventris Tamanuki, 1938b: 167 (Gaurotes) [HN]

virginea virginea Linnaeus, 1758: 398 (Leptura) E: AL AU

BH BU BY CR CT CZ EN FI FR GE GR HU IT LA LS

LT MD NR NT PL RO SK SL SV SZ ST UK YU

notaticollis Pic, 1916a: 10

sanguinaria Pic, 1917g: 4

thalassina Schrank, 1781a: 161 (Leptura)

MUST BE: virginea aemula Mannerheim, 1852b: 306 (Pachyta) E: CT ST A: ES FE HEI HUB JIL KZ MG NMO SHX WS ruficollis Solsky, 1871: 403 (Pachyta)

virginea kozhevnikovi Plavilstshikov, 1915c: 105 (Gaurotes) A: FE HEI JIL NC SC

komensis Tamanuki, 1938b: 167 (Gaurotes)

nigriventris Jureček, 1921: 25 (Gaurotes)

nigriventris Tamanuki, 1938b: 167 (Gaurotes) [HN]

sibirica Podan1, 1962: 236 (Gaurotes)

virginea thalassina Schrank, 1781a: 161 (Leptura) E: AU FR IT SL

virginea virginea Linnaeus, 1758: 398 (Leptura) E: AL AU

BE BH BU BY CR CT CZ EN FI FR GE GR HU IT LA

LS LT MD NR NT PL RO SK SL SV SZ ST UK YU

notaticollis Pic, 1916a: 10

sanguinaria Pic, 1917g: 4

violacea DeGeer, 1775: 144 (Leptura)

NOTE:

Pachyta (Carilia) virginea var. ruficollis Solsky, 1871: 403 was described from Baikal. Gaurotes sibirica Podan1, 1962 was described from "Ussuri" on the base of a specimen with black abdomen! The record of the species for Belgium [Drumont \& Grifnee, 2005] was overlooked.

\section{PAGE 127}

PRINTED:

incolumnis Heyden, 1886d: 273

MUST BE:

incolumis Heyden, 1886d: 273

\section{PAGE 127}

PRINTED:

debilis Kraatz, 1879d: 104 (Grammoptera) A: FE HEI JA NC SC TAI ZHE

MUST BE:

debilis Kraatz, 1879d: 104 (Grammoptera) A: FE HEI NC $\mathrm{SC}$ NOTE:

Pidonia debilis absent in Japan and Taiwan, as well as in Zhejiang prov. of China, replaced by closely related species.

86. PAGE 129

PRINTED:

subgenus Pidonia Mulsant, 1863: 570 type species Leptura lurida Fabricius, 1792

Pseudopidonia Pic, 1900s: 81 type species Pseudopidonia amurensis Pic, 1900

MUST BE:

subgenus Pidonia Mulsant, 1863: 570 type species Leptura lurida Fabricius, 1792 ...

subgenus Pseudopidonia Pic, 1900s: 81 type species Pseudopidonia amurensis Pic, 1900 $\cdots$

European Pidonia (s.str.) differs from East Asian $P$. (Pseudopidonia) by the unique combination of characters: $3^{\text {rd }}$ antennal joint about as long as $1^{\text {st }}$ and $2^{\text {nd }}$ combined or shorter; eyes with deap and distinct emargination.

\section{PAGE 129}

PRINTED:

alticollis Kraatz, 1879d: 103 (Grammoptera) A: CH FE

MUST BE:

alticollis Kraatz, 1879d: 103 (Grammoptera) A: CH FE $\underline{\text { NC }} \underline{\text { SC }}$ See Lee [1987]. 


\section{PAGE 129}

PRINTED:

amurensis Pic, 1900s: 81 (Pseudopidonia) A: FE JA JIL NC SC SHA

MUST BE:

amurensis Pic, 1900s: 81 (Pseudopidonia) A: FE JIL NC SC SHA

NOTE:

The species absent in Japan. The wrong record could be connected with wrong old identifications [Plavilstshikov, 1936] of $P$. amurensis males as $P$. signifera, as well as wrong synonyms published by Tsherepanov [1979]: "P. signifera $=$ P. amurensis".

\section{PAGE 129}

PRINTED:

chinensis Hayashi \& Villiers, 1985b: 17 A: CH

MUST BE:

chinensis Hayashi \& Villiers, 1985ạ: 17 A: CH

90. PAGE 129

PRINTED: NOTE:

rufiventris Plavilstshikov, 1932a: 87 (Pseudopidonia)

The name absent in the publication by Plavilstshikov [1932a]. It was introduced in the publication, which absent in the references (see note to the page 833): Plavilstshikov N.N. 1932: Lepturinen-Studien (Col., Cerambycidae). I. Časopis Československé Společnosti Entomologické 29: 87-88, 174-175.

\section{PAGE 131}

PRINTED:

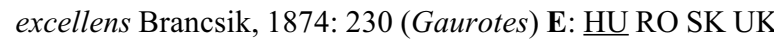
MUST BE:

excellens Brancsik, 1874: 230 (achyta) E: PL RO SK UK

\section{PAGE 134}

PRINTED:

vittatus Fischer von Waldheim, 1842: 19 (Toxotus) A: KZ XIN obliquus Motschulsky, 1845a: 86 (Toxotus) suvorovi Reitter, 1907a: 208 (Toxotus) turkestanicus Ganglbauer, 1889b: 280 (Toxotus)

MUST BE:

vittatus Fischer von Waldheim, 1842: 19 (Toxotus) [prevailing usage] A: KZ XIN

obliquus Motschulsky, 1845a: 86 (Toxotus)

suvorovi Plavilstshikov. 1936: 173 [unjustified emendation] suworowi Reitter, 1907a: 208 (Toxotus)

turkestanicus Ganglbauer, 1889b: 280 (Toxotus)

vittattus Fischer von Waldheim, 1842: 19 (Toxotus) [original spelling]

93. PAGE 141

PRINTED:

subgenus Necydalisca Plavilstshikov, 1936: 464 type species Necydalis ebenina Bates, 1884 (=Necydalis pennata Lewis, 1879)

MUST BE:

subgenus Necydalisca Plavilstshikov, 1936: 462 type species Necydalis ebenina Bates, 1884 (=Necydalis pennata Lewis, 1879)

NOTE:

The name ,ebenina Bates, 1884, Necydalis“ is absent in the "Index to species-group names": http://www.apollobooks.com/ PDF/CatPalColIndex_vol6.pdf

\section{PAGE 151}

PRINTED:

genus Leioderes L. Redtenbacher, 1849: 482 type species Leioderes kollari L. Redtenbacher, 1849

kollari L. Redtenbacher, 1849: 482 E: AL AU BH BU BY CR CT CZ FR GE GG GR HU IT LA LT MC MD NR RO PL SK SL SP ST SV SZ UK YU $\underline{\mathbf{A}: \text { TR }}$

MUST BE:

genus Leioderes L. Redtenbacher, 1849: 482 type species Leioderes kollari L. Redtenbacher, 1849

kollari L. Redtenbacher, 1849: 482 E: AL AU BH BU BY CR CT CZ FR GE GG GR HU IT LA LT MC MD NR RO PL SK SL SP ST SV SZ UK YU NOTE:

See Sama [2002: 72]: ,Old records from Syria and Asia Minor belong to L. tuerki Ganglbauer, 1885".

\section{PAGE 152}

PRINTED:

magnanii Sama \& Rapuzzi, 1999: 468 A: TR

MUST BE:

magnanii Sama \& Rapuzzi, 1999: 468 (Poecilium) A: TR

NOTE:

As the species was described in the genus Poecilium Fairmaire, 1864.

\section{PAGE 152}

PRINTED:

rufipes rufipes Fabricius, 1777: 232 (Callidium) E: AU BE BH BU CR CZ FR GE GR HU IT MD PL RO SK SL SP ST SZ UK YU A: TR

MUST BE:

rufipes rufipes Fabricius, 1777: 232 (Callidium) E: AU BE BH BU CR CZ FR GE GR HU IT MC MD PL RO SK SL SP ST SZ UK YU A: TR NOTE:

Two specimens of Phymatodes rufipes (Fabricius, 1777) were collected by L.Stefanov [personal message with a photo, 2010] near Elshani village, foot of Galicica Mt. 06.07.2010.

\section{PAGE 153}

PRINTED:

subgenus Phymatodellus Reitter, 1913a: 40 type species Callidium rufipes Fabricius, 1777

and

genus Poecilium Fairmaire, 1864a: 134 type species Leptura alni Linnaeus, 1767

Microcallidium Casey, 1912: 283 type species Callidium amoenus Say, 1823

Paraphymatodes Plavilstshikov, 1934a: 215 type species Callidium fasciatum Villers, 1789

Phymatoderus Reitter, 1913a: 39 [HN] type species Callidium pusillum Fabricius, 1787

Phymatodina Casey, 1912: 281 type species Phymatodes nitidus Casey, 1874

Pseudopoecilium Planet, 1924: 226 type species Callidium rufipes Fabricius, 1777

Reitteroderus Sama, 1991: 124 [unnecessary substitute name]

MUST BE:

subgenus Phymatodellus Reitter, 1913a: 40 type species Callidium rufipes Fabricius, 1777

Microcallidium Casey, 1912: 283 type species Callidium amoenus Say, 1823

Phymatodina Casey, 1912: 281 type species Phymatodes 
nitidus Casey, 1874

Pseudopoecilium Planet, 1924: 226 type species Callidium rufipes Fabricius, 1777 and

subgenus Poecilium Fairmaire, 1864a: 134 type species Leptura alni Linnaeus, 1767 and

subgenus Paraphymatodes Plavilstshikov, 1934a: 215 type species Callidium fasciatum Villers, 1789 and

subgenus Phymatoderus Reitter, 1913a: 39 type species Callidium pusillum Fabricius, 1787

Reitteroderus Sama, 1991: 124 [unnecessary substitute name]

NOTE:

The name Reitteroderus Sama, 1991 proposed as a replacement name for Phymatoderus Reitter, 1912 (regared as a junior homonym of Phymatoderus Dejean, 1837) was superficial [see Sama, 1999a], as Phymatoderus Dejean, 1837 was nomen nudum. Phymatoderus Reitter, 1912 is valid and Phymatoderus Reitter, $1912=$ Reitteroderus Sama, 1991.

\section{PAGE 154}

PRINTED:

pusillum pusillum Fabricius, 1787: 155 (Callidium) E: AU BE BH BU CR CZ FR GE GR HU IT MD NR PL RO SL SP SV SZ UK

Must Be: [in genus Phymatodes Subgenus Phymatoderus] pusillus pusillus Fabricius, 1787: 155 (Callidium) E: AU BE BH BU CR CZ FR GE GR HU IT MD NR PL RO SL SP SV SZ TR UK A: TR See: Özdikmen [2008a: 43-44].

99. PAGES 158-159

PRINTED:

genus Cerambyx Linnaeus, 1758: 388 type species Cerambyx cerdo Linnaeus, 1758

Hamaticherus Dejean, 1821: 105 type species Cerambyx heros Scopoli, 1763 (= Cerambyx cerdo Linnaeus, 1758) Hammatocerus Gistel, 1848a: 130 [unjustified emendation] [HN]

Microcerambyx Mikšić \& Georgijevic, 1973: 22 type species Cerambyx scopolii Fuessly, 1775 apiceplicatus Pic, 1941b: 2 A: IQ

carinatus Küster, 1845a: 46 (Hammaticherus) E: BH BU CR GR IT MA MC YU A: TR

landrieui Pic, 19271: 158 minor Pic, 1926d: 13

cerdo cerdo Linnaeus, 1758: 392 E: AB AL AR AU BE BH BU BY CR CT CZ FR GBi GE GG GR HU IR IT LA LU MA MC MD NL PL PT RO SK SL SP ST SZ TR UK YU N: MO A: IN IQ IS JO LE SY TR

acuminatus Motschulsky, 1853: 79

heros Scopoli, 1763: 51

iranicus Heyrovsk1, 1951: 156

klinzigi Podan1, 1964c: 88

manderstjaernae Mulsant \& Godart, 1855a: 180 pfisteri Stierlin, 1864: 152

cerdo mirbeckii P. H. Lucas, 1842: 184 (Hamaticherus) N: AG MO TU tunisicus Pic, 1891b: 18 [DA]

dux Faldermann, 1837: 264 (Hammaticherus) E: AB AR BU GG MC ST UK A: IN IS JO LE SY intricatus Fairmaire, 1848: 167 (Hammaticherus) nodosus Mulsant \& Rey, 1863: 144 orientalis Küster, 1845a: 45 (Hammaticherus)

thirkii Küster, 1845a: 47 (Hammaticherus)

elbursi Jureček, 1924a: 47 A: IN

heinzianus Demelt, 1976: 65 E: GG

miles Bonelli, 1812: 178 E: AB AL AR AU BH BU CR FR GG GR HU IT MC PT RO SK SL SP ST SZ TR UK YU A: TR militaris Latreille, 1829: 116 (Hamaticherus)

multiplicatus Motschulsky, 1860a: 142 E: AB A: IN elegans Dohrn, 1873: 74

nodulosus Germar, 1817: 220 E: AB AL AR BH BU CR GG GR IT MA MC RO SL ST TR UK YU A: CY LE SY nodicornis Küster, 1845a: 43 (Hammaticherus)

paludivagus $\mathrm{P}$. H. Lucas, 1842: 185 (Hammaticherus) N: AG TU

scopolii Fuessly, 1775: 12 E: AB AL AR AU BE BH BU BY CR CT CZ DE EN FR GB GE GG GR HU IR IT LA LT LU MC MD NL NR NT PL PT RO SK SL SP ST SV SZ TR UK YU A: SY TR

helveticus Stierlin, 1878b: 442

nitidus Pic, 1892s: cxi [= 1893d: 417]

piceus Geoffroy, 1785: 74

welensii Küster, 1845a: 44 (Hammaticherus) E: AL AB BH BU CR FR GG GR HU IT PT RO SK SL SP UK YU A: CY IN IS JO LE SY TR

centurio Czwalina, 1891: 99 velutinus Brullé, 1832: 252 [HN]

MUST BE:

genus Cerambyx Linnaeus, 1758: 388 type species Cerambyx cerdo Linnaeus, 1758

subgenus Cerambyx Linnaeus, 1758: 388 type species Cerambyx cerdo Linnaeus, 1758

Hamaticherus Dejean, 1821: 105 type species Cerambyx heros Scopoli, 1763 (= Cerambyx cerdo Linnaeus, 1758) Hammatocerus Gistel, 1848a: 130 [unjustified emendation] [HN]

apiceplicatus Pic, 1941b: 2 A: IQ

carinatus Küster, 1845a: 46 (Hammaticherus) E: BH BU CR GR IT MA MC YU A: TR

landrieui Pic, 19271: 158

minor Pic, 1926d: 13

cerdo cerdo Linnaeus, 1758: 392 E: AB AL AR AU BE BH BU BY CR CT CZ FR GBi GE GG GR HU IR IT LA LU MA MC MD NL PL RO SK SL ST SZ TR UK YU N: MO A: IN IQ IS JO LE SY TR

acuminatus Motschulsky, 1853: 79

heros Scopoli, 1763: 51

iranicus Heyrovsk1, 1951: 156

klinzigi Podan1, 1964c: 88

manderstjaernae Mulsant \& Godart, 1855a: 180

cerdo mirbeckii P.H. Lucas, 1842: 184 (Hamaticherus) N: AG MO PT SP TU

tunisicus Pic, 1891b: 18 [DA]

cerdo pfisteri Stierlin, 1864: 152 E: IT GR [See Villiers, 1978: 302]

dux Faldermann, 1837: 264 (Hammaticherus) E: AB AR BU GG MC ST UK A: IN IS JO LE SY

intricatus Fairmaire, 1848: 167 (Hammaticherus)

nodosus Mulsant \& Rey, 1863: 144

orientalis Küster, 1845a: 45 (Hammaticherus)

thirkii Küster, 1845a: 47 (Hammaticherus)

heinzianus Demelt, 1976: 65 E: GG

miles Bonelli, 1812: 178 E: AB AL AR AU BH BU CR FR GG GR HU IT MC PT RO SK SL SP ST SZ TR UK YU A: TR militaris Latreille, 1829: 116 (Hamaticherus)

nodulosus Germar, 1817: 220 E: AB AL AR BH BU CR GG GR IT MA MC RO SL ST TR UK YU A: CY LE SY 
nodicornis Küster, 1845a: 43 (Hammaticherus)

welensii Küster, 1845a: 44 (Hammaticherus) E: AL AB BH BU CR FR GG GR HU IT PT RO SK SL SP UK YU A: CY IN IS JO LE SY TR

centurio Czwalina, 1891: 99 velutinus Brullé, 1832: 252 [HN]

subgenus Microcerambyx Mikšić \& Georgijevic, 1973: 22 type species Cerambyx scopolii Fuessly, 1775 elbursi Jureček, 1924a: 47 A: IN

multiplicatus Motschulsky, 1860a: 142 E: AB A: IN elegans Dohrn, 1873: 74

paludivagus $\mathrm{P}$. H. Lucas, 1842: 185 (Hammaticherus) N: AG TU

scopolii Fuessly, 1775: 12 E: AB AL AR AU BE BH BU BY CR CT CZ DE EN FR GB GE GG GR HU IR IT LA LT LU MC MD NL NR NT PL PT RO SK SL SP ST SV SZ TR UK YU A: SY TR

helveticus Stierlin, $1878 \mathrm{~b}$ : 442

nitidus Pic, 1892s: cxi [= 1893d: 417]

piceus Geoffroy, 1785: 74

100. PAGE 176

PRINTED:

genus Plagionotus Mulsant, 1842b: 1 type species Leptura detrita Linnaeus, 1758

Echinocerus Mulsant, 1862: 143 type species Cerambyx floralis Pallas, 1773

Neoplagionotus Kasatkin, 2005: 51 type species Clytus bobelayei Brullé, 1832

Paraplagionotus Kasatkin, 2005: 51 [unnecessary RN]

Platynotus Mulsant, 1839: 71 [HN] type species Leptura detrita Linnaeus, 1758

andreui Fuente, 1908a: 21 E: SP

marcae López-Colón, 1997: 219 [incorrect orig. spelling] marcaorum López-Colón, 1997: 219

marcorum Vives, 2000: 190 [incorrect emendation]

arcuatus Linnaeus, 1758: 399 (Leptura) E: AL AR AU BE BH BU BY CR CT CZ DE EN FI FR GE GG GR HU IR IT LA LT LU MD NL NR NT PL PT RO SK SL SP ST SV SZ TR UK YU N: AG MO TU A: IN KI KZ SY TR apicalis Hampe, 1863: 289 (Clytus)

buyssoni Dauphin, 1924: 42

interrupteconnatus G. Schmidt, 1951: 16

lunatus Fabricius, 1782: 500 (Callidium)

martialis Pic, $1918 \mathrm{~d}: 15$

milliati Pic, 1934e: 20

multiinterruptus Pic, 1933d: 6

pagnioni Pic, 1925d: 10

reichei J. Thomson, 1861: 220 (Plagyonotus)

salicis Schrank, 1798: 677 (Clytus)

stauropolibus Pic, 1915e: 7

bartholomei Motschulsky, 1860a: 142 (Clytus) E: AB A: IN admirabilis Heyden, 1878: 314 (Clytus) bisbifasciatus Pic, 1915f: 13 A: YUN

bobelayei Brullé, 1832: 253 (Clytus) E: AB AL AR BU GG GR MC RO ST TR UK A: IN IS JO SY TM TR luristanicus Pic, 1911a: 6

mouzafferi Pic, 1905g: 114

persicus Pic, 1951a: speciosus Adams, 1817: 309 (Callidium) [HN]

christophi Kraatz, 1879d: 108 (Clytus) A: ANH FE HEB HEI HEN HUB JA JIL LIA NC SC SHA

detritus Linnaeus, 1758: 399 (Leptura) E: AB AL AR AU BE BH BU BY CR CT CZ EN FR GE GG GR HU IT LA LT MD NL NT PL PT RO SK SL SP ST SV SZ TR UK YU A: KZ SY TR africaeseptentrionalis Tippmann, 1952a: 143

anticereductus G. Schmidt, 1951: 14

convertini L. Petagna, 1819: 38 (Callidium)

interrupteconnatus G. Schmidt, 1951: 16

obscurebasalis Pic, 1942b: 2

rufescens Pic, 1891b: 24

uralensis Tippmann, 1952a: 144

floralis Pallas, 1773: 724 (Cerambyx) E: AB AL AR AU BH

BU CR CT CZ FR GE GG GR HU IT LA LT MC MD NT PL RO SK SL SP ST SZ TR UK YU A: ES IN IS JO KI KZ TD TM TR UZ WS XIN

abruptus Kraatz, 1871b: 408 (Clytus)

araratensis Pic, 1901b: 11 (Clytus)

arcuatus Scopoli, 1772: 97 (Stenocorus)

armeniacus Reitter, 1890c: 213

aulicus Laicharting, 1784: 103 (Clytus)

basicornis Reitter, 1890c: 213

clermonti Pic, $1913 \mathrm{c}$ : 121

controversus Schrank, 1798: 679 (Clytus)

fasciatus Herbst, 1784: 98 (Callidium)

indicus Gmelin, 1790: 1856 (Callidium)

massiliensis Pic, 1951a: 1 (Plagionotus)

pilifer Reitter, 1890c: 213

pruinosus Kraatz, 1871b: 409 (Clytus)

variabilis Motschulsky, 1860a: 144 [= 1860c: 305$]$ (Clytus) zebra Dalman, 1817b: 194 (Clytus)

lugubris Ménétriés, 1832: 229 (Clytus) E: AB AR ST A: IN TM flavicornis Pic, 1898b: 19

henoni Pic, 1933d: 6

lenkoranus Pic, 1933d: 6

pulcher Blessig, 1872: 184 (Clytus) A: FE HEB HEI JA JIL NC NIN SC SHA SHX

lignatorum Thieme, 1881: 100 (Clytus)

maculithorax Pic, 1904d: 15

scalaris Brullé, 1832: 254 (Clytus) E: GR IT N: AG MO TU interruptus Dayrem, 1928: 77

siculus Laporte \& Gory, 1836: 46 (Clytus)

vivesi López-Colón, 1997: 221

MUST BE:

genus Echinocerus Mulsant, 1862: 143 type species Cerambyx floralis Pallas, 1773

Paraplagionotus Kasatkin, 2005: 51 [unnecessary RN]

floralis Pallas, 1773: 724 (Cerambyx) E: AB AL AR AU BH BU CR CT CZ FR GE GG GR HU IT LA LT MC MD NT PL RO SK SL SP ST SZ TR UK YU A: ES IN IS JO KI KZ TD TM TR UZ WS XIN abruptus Kraatz, 1871b: 408 (Clytus) araratensis Pic, 1901b: 11 (Clytus) arcuatus Scopoli, 1772: 97 (Stenocorus) armeniacus Reitter, 1890c: 213 (Plagionotus) aulicus Laicharting, 1784: 103 (Clytus) basicornis Reitter, 1890c: 213 (Plagionotus) clermonti Pic, 1913c: 121 (Plagionotus) controversus Schrank, 1798: 679 (Clytus) fasciatus Herbst, 1784: 98 (Callidium) indicus Gmelin, 1790: 1856 (Callidium) massiliensis Pic, 1951a: 1 (Plagionotus) pilifer Reitter, 1890c: 213 (Plagionotus) pruinosus Kraatz, 1871b: 409 (Clytus) variabilis Motschulsky, 1860a: 144 [= 1860c: 305] (Clytus)

zebra Dalman, 1817b: 194 (Clytus)

and

genus Neoplagionotus Kasatkin, 2005: 51 type species Clytus bobelayei Brullé, 1832

andreui Fuente, 1908a: 21 (Plagionotus) E: SP 
marcae López-Colón, 1997: 219 (Plagionotus) [incorrect orig. spelling]

marcaorum López-Colón, 1997: 219 (Plagionotus) marcorum Vives, 2000: 190 (Plagionotus) [incorrect emendation]

bobelayei Brullé, 1832: 253 (Clytus) E: AB AL AR BU GG GR MC RO ST TR UK A: IN IS JO SY TM TR

luristanicus Pic, 1911a: 6 (Plagionotus) mouzafferi Pic, 1905g: 114 (Plagionotus) persicus Pic, 1951a: 1 (Plagionotus) speciosus Adams, 1817: 309 (Callidium) [HN]

scalaris Brullé, 1832: 254 (Clytus) E: GR IT N: AG MO TU interruptus Dayrem, 1928: 77 (Plagionotus) siculus Laporte \& Gory, 1836: 46 (Clytus) vivesi López-Colón, 1997: 221 (Plagionotus) and

genus Plagionotus Mulsant, 1842b: 1 type species Leptura detrita Linnaeus, 1758

Plagyonotus J. Thomson, 1861: 220 [unjustified emendation]

Platynotus Mulsant, 1839: 71 [HN] type species Leptura detrita Linnaeus, 1758

arcuatus Linnaeus, 1758: 399 (Leptura) E: AL AR AU BE BH BU BY CR CT CZ DE EN FI FR GE GG GR HU IR IT LA LT LU MD NL NR NT PL PT RO SK SL SP ST SV SZ TR UK YU N: AG MO TU A: IN KI KZ SY TR apicalis Hampe, 1863: 289 (Clytus)

buyssoni Dauphin, 1924: 42

interrupteconnatus G. Schmidt, 1951: 16

lunatus Fabricius, 1782: 500 (Callidium)

martialis Pic, 1918d: 15

milliati Pic, 1934e: 20

multiinterruptus Pic, 1933d: 6

pagnioni Pic, $1925 \mathrm{~d}$ : 10

reichei J. Thomson, 1861: 220 (Plagyonotus)

salicis Schrank, 1798: 677 (Clytus)

stauropolibus Pic, $1915 \mathrm{e}: 7$

bartholomei Motschulsky, 1860a: 142 (Clytus) E: AB A: IN admirabilis Heyden, 1878: 314 (Clytus) bisbifasciatus Pic, 1915f: 13 A: YUN

christophi Kraatz, 1879d: 108 (Clytus) A: ANH FE HEB HEI HEN HUB JA JIL LIA NC SC SHA

detritus Linnaeus, 1758: 399 (Leptura) E: AB AL AR AU BE BH BU BY CR CT CZ EN FR GE GG GR HU IT LA LT MD NL NT PL PT RO SK SL SP ST SV SZ TR UK YU A: KZ SY TR

africaeseptentrionalis Tippmann, 1952a: 143

anticereductus $\mathrm{G}$. Schmidt, 1951: 14

convertini L. Petagna, 1819: 38 (Callidium)

interrupteconnatus G. Schmidt, 1951: 16

obscurebasalis Pic, 1942b: 2

rufescens Pic, 1891b: 24

uralensis Tippmann, 1952a: 144

lugubris Ménétriés, 1832: 229 (Clytus) E: AB AR ST A: IN TM flavicornis Pic, 1898b: 19

henoni Pic, 1933d: 6

lenkoranus Pic, 1933d: 6

pulcher Blessig, 1872: 184 (Clytus) A: FE HEB HEI JA JIL NC NIN SC SHA SHX

lignatorum Thieme, 1881: 100 (Clytus)

maculithorax Pic, 1904d: 15

101. PAGE 177

PRINTED:

detritus Linnaeus, 1758: 399 (Leptura) E: AB AL AR AU BE BH BU BY CR CT CZ EN FR GE GG GR HU IT LA LT
MD NL NT PL PT RO SK SL SP ST SV SZ TR UK YU A: KZ SY TR

africaeseptentrionalis Tippmann, 1952a: 143

anticereductus G. Schmidt, 1951: 14

convertini L. Petagna, 1819: 38 (Callidium)

interrupteconnatus G. Schmidt, 1951: 16

obscurebasalis Pic, 1942b: 2

rufescens Pic, 1891b: 24

uralensis Tippmann, 1952a: 144

MUST BE:

detritus caucasicola Plavilstshikov, 1936: 435 E: AB AR GG ST A: SY TR

detritus detritus Linnaeus, 1758: 399 (Leptura) E: AB AL AR AU BE BH BU BY CR CT CZ EN FR GE GG GR HU IT LA LT MD NL NT PL PT RO SK SL SP ST SV SZ TR UK YU A: KZ SY TR

africaeseptentrionalis Tippmann, 1952a: 143

anticereductus $\mathrm{G}$. Schmidt, 1951: 14

convertini L. Petagna, 1819: 38 (Callidium)

interrupteconnatus G. Schmidt, 1951: 16

obscurebasalis Pic, 1942c: 2

rufescens Pic, 1891b: 24

uralensis Tippmann, 1952a: 144

NOTE:

Plagionotus detritus caucasicola Plavilstshikov, 1940 was described with two taxonomical rank in one page [435] "form" and "morph": ["... evidently it is not more than poorly pronounced geographical form; we separate it now as a morph (m. caucasicola n. fig. 263)."'] [in Russian]. So, it is available name, as its geographical character was stated.

102. PAGE 177

PRINTED:

obscurebasalis Pic, 1942ㅁ: 2

MUST BE:

obscurebasalis Pic, 1942c: 2

103. PAGE 180

PRINTED:

genus Turanoclytus Sama, 1994e: 325 type species Clytus namanganensis Heyden, 1885

ilamensis campadellii Sama \& Rapuzzi, 2003: 92 E: AB A: IN

raghidae Sama \& Rapuzzi, 2000: 14 A: IS LE

MUST BE:

genus Turanoclytus Sama, 1994e: 325 type species Clytus namanganensis Heyden, 1885

ilamensis campadellii Sama \& Rapuzzi, 2003: 92 (Xylotrechus) E: AB A: IN

raghidae Sama \& Rapuzzi, 2000: $14 \underline{\text { (Xylotrechus) }}$ A: IS LE NOTE:

But in fact Turanoclytus must be regarded as a subgenus of Xylotrechus! Besides X. ilamensis, X. raghidae and X. sieversi have no connection to Turanoclytus and must be placed in a special subgenus.

104. PAGES 180 AND 183

PRINTED:

p. 180

subgenus Kostiniclytus Danilevsky, 2009: 211 type species:

Xylotrechus zaisanicus Plavilstshikov, 1940

arnoldii Kostin, 1974: 647 A: KZ 
medvedevi Danilevsky, 2009: 216 A: MG

zaisanicus Plavilstshikov, 1940a: 354 A: KZ p. 183

yanoi Gressitt, 1934: 164 A: BEI JA NMO SC ORR pekingensis Pic, 1939b: 3

zaisanicus Plavilstshikov, 1940a: 354 A: KZ

arnoldii Kostin, 1974: 647

zebratus Matsushita, 1938a: 93 A: JA

MUST BE:

subgenus Kostiniclytus Danilevsky, 2009: 211 type species: Xylotrechus zaisanicus Plavilstshikov, 1940

arnoldii Kostin, 1974: 647 A: KZ

medvedevi Danilevsky, 2009: 216 A: MG

zaisanicus Plavilstshikov, 1940a: 354 A: KZ and

yanoi Gressitt, 1934: 164 A: BEI JA NMO SC ORR pekingensis $\mathrm{Pic}, 1939 \mathrm{~b}: 3$

zebratus Matsushita, 1938a: 93 A: JA

\section{PAGE 185}

PRINTED:

sericeus Fabricius, 1787: 152 (Callidium) E: AB AL AR BH CR FR GG GR MA PT SP ST SZ UK YU N: AG EG LB MO TU A: CY IN IQ IS JO TM TR

\section{MUST BE:}

sericeus Fabricius, 1787: 152 (Callidium) E: AB AL AR BH CR FR GG GR MA MC PT SP ST SZ UK YU N: AG EG LB MO TU A: CY IN IQ IS JO TM TR NOTE:

A female of Hesperophanes sericeus (Fabricius, 1787) was collected by L.Stefanov [personal message with a photo, 2010] in Skopje 25.08.2010.

106. PAGES 188, 190 AND 191

PRINTED:

genus Glaphyra Newman, 1840b: 19 type species Glaphyra semiusta Newman, 1840 and

genus Molorchus Fabricius, 1792b: 356 type species Necydalis minor Linnaeus, 1758

Caenoptera C. G. Thomson, 1859: 150 type species Necydalis minor Linnaeus, 1758 and

genus Nathrioglaphyra Sama, 1995a: 383 type species Molorchus heptapotamicus Plavilstshikov, 1940

MUST BE:

genus Molorchus Fabricius, 1792b: 356 type species Necydalis umbellatarum Schreber, 1759 Glaphyra Newman, 1840b: 19 type species Glaphyra semiusta Newman, 1840

subgenus Caenoptera C. G. Thomson, 1859: 150 type species Necydalis minor Linnaeus, 1758 and

subgenus Molorchus Fabricius, 1792b: 356 type species Necydalis umbellatarum Schreber, 1759 and

subgenus Nathrioglaphyra Sama, 1995a: 383 type species Molorchus heptapotamicus Plavilstshikov, 1940 NOTE:

The type species of genus Molorchus Fabricius, 1792 is Necydalis umbellatarum Schreber, 1759 [Bousquet, 2008], but not Necydalis minor Linnaeus, 1758, as it was recently accepted by several authors [Sama, 2002; Niisato, 2007 and others]. So, Caenoptera C. G. Thomson, 1859: 150 type species Necydalis minor Linnaeus, 1758 is valid, as it was traditionally accepted before [Plavilstshikov, 1940; Heyrovsk1, 1955 and others]; and Molorchus Fabricius, 1792 = Glaphyra Newman, 1840 [Linsley, 1963]. In fact both taxa Caenoptera and Molorchus must be regarded as subgenera of one genus, as it was generally accepted before the publication by A. Villiers [1978], who inadequately raised many subgenera to genus level.

107. PAGE 197 and 199

PRINTED:

genus Purpuricenus Dejean, 1821: 105 type species Cerambyx kaehleri Linnaeus, 1758

subgenus Purpuricenus Dejean, 1821: 105 type species Cerambyx kaehleri Linnaeus, 1758

and

subgenus Sternoplistes Guérin-Méneville, 1844: 224 type species Sternoplistes temminckii Guérin-Méneville, 1844 NOTE:

The current division of Purpuricenus in two subgenera is definitely wrong. It is connected with the common fact, that western authors did not know eastern species, and eastern authors did not know western species good enouph.

The main distinguishing characters of Sternoplistes (central swelling on the base of pronotum and tubercles on the sternal processes of pro- and metathorax) can be seen in certain species of Purpuricenus s. str. (talyshensis, deyrollei, desfontainei), while pronotal swelling in P.(S.) lituratus nearly indistinct, and that is why it was regarded as Purpuricenus s. str. by Gressitt [1951a] and many other authors.

In fact Purpuricenus is quite artificial group, consisting of several good genera joined together only because of contrast black-red color. Now it is better to treat the genus without any subgenera, as it was done for example by Plavilstshikov [1940], because the current set of species for each of two is accidental.

108. PAGE 198

PRINTED:

caucasicus caucasicus T. Pic, 1902: 27 E: AB AR GG ST TR caucasicus renyvonae Sláma, 2001: 225 E: BU CR MC YU

$\mathrm{UK}$

baeckmanni Danilevsky, 2007c: 38

and

graecus Sláma, 1993: 56 E: GR

MUST BE:

caucasicus baeckmanni Danilevsky, 2007c: 38 [DA] E: UK caucasicus caucasicus T. Pic, 1902: 27 E: AB AR GG ST TR caucasicus graecus Sláma, 1993: 56 [DA] E: GR

caucasicus renyvonae Sláma, 2001: 225 [DA] E: BU CR MC YU NOTE:

All four taxa are very close to each other morphologically, but their areas are distant and geographically isolated. Each taxon is known in a small number of specimens, and individual variability of each populations is not clear, so real taxonomical relations inside the group need further invsetigations.

109. PAGE 198

PRINTED:

dalmatinus Sturm, 1843: 353 E: BH BU CR GR IT MC SL

UK A: IS JO LE SY TR

MUST BE:

dalmatinus Sturm, 1843: 353 E: BH BU CR GR IT MC SL A: IS JO LE SY TR

NOTE:

No records of Purpuricenus dalmatinus for Ukraine were ever known. 


\section{PAGES 203-205}

PRINTED:

genus Callimus Mulsant, 1846: [5] type species Callimus bourdini Mulsant, 1846 (= Saperda angulata Schrank, 1789) and

genus Lampropterus Mulsant, 1862: 214 type species Necydalis femoratus Germar, 1824 and

genus Procallimus Pic, 1907b: 7 type species Callimus egregius Mulsant \& Rey, 1863

MUST BE:

genus Callimus Mulsant, 1846: [5] type species Callimus bourdini Mulsant, 1846 (= Saperda angulata Schrank, 1789)

subgenus Callimus Mulsant, 1846: [5] type species Callimus bourdini Mulsant, 1846 (= Saperda angulata Schrank, 1789)

and

subgenus Lampropterus Mulsant, 1862: 214 type species Necydalis femoratus Germar, 1824 and

subgenus Procallimus Pic, 1907b: 7 type species Callimus egregius Mulsant \& Rey, 1863

NOTE: level.

The differences between all three taxa are of subgeneric

\section{PAGE 205}

PRINTED:

rufus geniculatus Kraatz, 1863: 104 E: AL BU CR GR MC RO SL TR YU A: IN

rufus rufus Linnaeus, 1767: 642 (Necydalis) E: AB AR AU BE BH BU CR CZ FR GE GG HU IT LU MA MD NL PL SK SL SP ST SZ UK N: CI (Gran Canaria) A: TM attenuatus Geoffroy, 1785: 84 (Leptura)

rufus syriacus Pic, 1892c: 22 A: IS LE SY TR

MUST BE:

rufus geniculatus Kraatz, 1863: 104 E: AB AL AR BU CR GG GR MC RO SL TR YU

rufus rufus Linnaeus, 1767: 642 (Necydalis) E: AU BE BH BU CR CZ FR GE GG HU IT LU MA MD NL PL SK SL SP ST SZ UK N: CI (Gran Canaria) attenuatus Geoffroy, 1785: 84 (Leptura)

rufus syriacus Pic, 1892c: 22 A: IS LE SY TR rufus transcaspicus Lazarev, 2008: 132 A: TM IR NOTE:

Stenopterus rufus geniculatus (because of black hind apices of hind femora) is similarly poor subspecies as many others accepted in Cerambycidae (Rutpela maculata nigricornis, Lepturalia nigripes rifipennis, Vadonia bipunctata mulsantiana and so on), with many transitional populations and many typically light (as in nominative subspecies) specimens in about each population. Any way the percentage of dark hind femora specimens in Transcaucasie (and in Crimea) is about same as in Bulgaria.

The reference to Lazarev [2008] was missing.

112. PAGES 213-214

PRINTED:

genus Agapanthia Audinet-Serville, 1835a: 35 type species Cerambyx cardui Linnaeus, 1767

subgenus Agapanthia Audinet-Serville, 1835a: 35 type species Cerambyx cardui Linnaeus, 1767

Eucrius Gistel, 1856: 376 type species Cerambyx cardui Linnaeus, 1767

Homoblephara Pesarini \& Sabbadini, 2004b: 128 type species Saperda maculicornis Gyllenhal, 1817

Segmentaria Gistel, 1848a: viii [unnecessary substitute name]

Smaragdula Pesarini \& Sabbadini, 2004b: 128 type species Saperda violacea Fabricius, 1775

and

subgenus Epoptes Gistel, 1857b: 93 type species Lamia asphodeli Latreille, 1804

Agapanthiella Pesarini \& Sabbadini, 2004b: 126 type species Cerambyx villosoviridescens DeGeer, 1775 Agapanthoplia Pesarini \& Sabbadini, 2004b: 122 type species Agapanthia coeruleipennis Frivaldszky, 1878 Amurobia Pesarini \& Sabbadini, 2004b: 128 type species Agapanthia amurensis Kraatz, 1879

Chionosticta Pesarini \& Sabbadini, 2004b: 122 type species Agapanthia niveisparsa Holzschuh, 1981

Drosotrichia Pesarini \& Sabbadini, 2004b: 126 type species Saperda annularis Olivier, 1795

Stichodera Pesarini \& Sabbadini, 2004b: 126 type species Saperda irrorata Fabricius, 1787

Synthapsia Pesarini \& Sabbadini, 2004b: 121 type species Saperda kirbyi Gyllenhal, 1817 NOTE:

Most of new names by Pesarini \& Sabbadini [2004b] must be accepted as valid subgenera names (with a single exception of Agapanthiella Pesarini \& Sabbadini, 2004b) subgenus Agapanthoplia Pesarini \& Sabbadini, 2004b: 122 type species Agapanthia coeruleipennis Frivaldszky, 1878 subgenus Amurobia Pesarini \& Sabbadini, 2004b: 128 type species Agapanthia amurensis Kraatz, 1879

subgenus Chionosticta Pesarini \& Sabbadini, 2004b: 122 type species Agapanthia niveisparsa Holzschuh, 1981

subgenus Drosotrichia Pesarini \& Sabbadini, 2004b: 126 type species Saperda annularis Olivier, 1795

subgenus Homoblephara Pesarini \& Sabbadini, 2004b: 128 type species Saperda maculicornis Gyllenhal, 1817

subgenus Smaragdula Pesarini \& Sabbadini, 2004b: 128 type species Saperda violacea Fabricius, 1775

subgenus Stichodera Pesarini \& Sabbadini, 2004b: 126 type species Saperda irrorata Fabricius, 1787

subgenus Synthapsia Pesarini \& Sabbadini, 2004b: 121 type species Saperda kirbyi Gyllenhal, 1817

113. PAGE 229

PRINTED:

agapanthina kani Hayashi, 1976: 15 A: JA

MUST BE:

kani Hayashi, 1976: 15 A: JA

NOTE:

Asaperda kani Hayashi, 1976 is a species as it is sympatric with $A$. agapanthina Bates, 1873 [according to the personal message by N.Ohbayashi, 2010].

\section{PAGES 242}

PRINTED:

tibiale Jakovlev, 1889: 250 A: KI XIN

MUST BE:

tibiale Jakovlev, 1889: 250 (Compsodorcadion) A: KI XIN

\section{PAGES 242}

PRINTED:

fulvum erythropteron Fischer von Waldheim, 1823: pl. L E:

AL BH BU CR MC MD RO PL UK YU

MUST BE:

fulvum erythropterum Fischer von Waldheim, 1823: Tab. L

E: AL BH BU CR MC MD RO PL UK YU 


\section{PAGES 243}

\section{PRINTED:}

albosuturale Breuning, 1946: 115 E: AL GR

MUST BE:

albosuturale Breuning, 1946: 115 E: AL GR $\underline{\text { MC }}$

NOTE:

Several specimens were collected in Macedonia near Ochrid lake by F.Tippmann in 1953 (my collection); several specimens were observed in Galičica National Park by L.Stefanov in 2010.

\section{PAGES 245}

PRINTED:

cinerarium cinerarium Fabricius, 1787: 140 (Lamia) E: CT MD ST UK

macropoides Plavilstshikov, 1932b: 183

perroudi Pic, 1942a: 2

euxinum Suvorov, 1915: 119

tricolor Fischer von Waldheim, 1805: 15 (Lamia)

MUST BE (SEE NOTE 5 TO PAGES 44-45):

cinerarium cinerarium Fabricius, 1787: 140 (Lamia) E: CT

MD ST UK

macropoides Plavilstshikov, 1932b: 183

perroudi Pic, 1942b: 2

tricolor Fischer von Waldheim, 1805: 15 (Lamia)

118. PAGES 246 AND 249

PRINTED:

drusum Chevrolat, 1870: 84 A: IS LE

and

libanoticum Kraatz, 1873a: 100 A: LE SY

apicale Chevrolat, 1873: 205 [HN]

perrini Fairmaire, 1881: 88

tarabuliense Ganglbauer, 1889d: 481

MUST BE:

drusum Chevrolat, 1870: 84 A: IS LE SY

apicale Chevrolat, 1873: 205 [HN]

libanoticum Kraatz, 1873a: 100

perrini Fairmaire, 1881: 88

tarabuliense Ganglbauer, 1889d: 481

NOTE:

According to Sama et al. [2010: 27] Dorcadion drusum Chevrolat, 1870 = Dorcadion libanoticum Kraatz, 1873.

\section{PAGE 248}

PRINTED:

indutum indutum Faldermann, 1837: 276 E: AB AR pulchrum Pic, 1908i: 58

indutum nigrosuturatum Reitter, 1897b: 236 E: AR griseipenne Breuning, 1943b: 92

MUST BE:

indutum Faldermann, 1837: 276 E: AB AR pulchrum Pic, 1908i: 58 ...

nigrosuturatum Reitter, 1897b: 236 E: AR griseipenne Breuning, 1943b: 92 NOTE:

Both taxa can not be regarded as subspecies, because represent two marginal forms in a long line of Alpine vicariant species along Sevan Ridge with several species in between (D. semilucens, D. cineriferum). Dark D. nigrosuturatum Reitter, $1897 \mathrm{~b}$ is the most north-western one distributed northwards Tzovagiuh at the northern most part of Sevan Lake. Light D. indutum Faldermann, 1837 is the most south-eastern one - distributed near Goris and in Karabakh.
120. PAGE 252

PRINTED:

sareptanum kubanicum Plavilstshikov, 1934d: 120 E: ST

MUST BE (SEE NOTE 5 TO PAGES 44-45):

sareptanum euxinum Suvorov, 1915 E: ST UK

kubanicum Plavilstshikov, 1934d: 120

121. PAGE 255

PRINTED:

cephalotes Jakovlev, 1889: 252 A: KZ

MUST BE:

cephalotes Jakovlev, 1889: 252 (Compsodorcadion) A: KZ

122. PAGE 264

PRINTED:

Ibidimorphum Blessig, 1872: 191 type species Ibidimorphum octopustulatum Motschulsky, 1860

MUST BE:

Ibidimorphum Motschulsky 1860:152 type species Ibidimorphum octopustulatum Motschulsky, 1860

123. PAGE 267

PRINTED:

clarus clarus Pascoe, 1859: 44 A: ANH FE FUJ GUI HEB

HEN HUB HUN JA JIA JIX NC SC SCH SHN TAI ZHE MUST BE:

clarus Pascoe, 1859: 44 A: ANH FE FUJ GUI HEB HEN

HUB HUN JA JIA JIX NC SC SCH SHN TAI ZHE NOTE:

«clarus clarus» is a rudiment of early version with «clarus subobliteratus».

124. PAGE 267

PRINTED:

textor Linnaeus, 1758: 239 (Cerambyx) E: AB AL AR AU

BE BH BU BY CR CT CZ DE EN FI FR GB GE GG GR

HU IR IT LA LT LU MC MD NL NR NT PL RO SK SL

SP ST SV SZ UK YU A: ES FE HEB HEI JA JIL KZ MG NC NMO SC SHN TAI WS XIN

MUST BE:

textor Linnaeus, 1758: 392 (Cerambyx) E: AB AL AR AU

BE BH BU BY CR CT CZ DE EN FI FR GB GE GG GR

HU IR IT LA LT LU MC MD NL NR NT PL RO SK SL

SP ST SV SZ UK YU A: ES FE HEB HEI JA JIL KZ MG

NC NMO SC SHN TAI WS XIN

125. PAGE 268

PRINTED:

asper asper Sulzer, 1776: 44 (Cerambyx) E: AL CR FR GR

IT SP SZ YU

ganglbaueri Reitter, 1894b: 44

MUST BE:

asper asper Sulzer, 1776: 44 (Cerambyx) E: AL CR FR GR

IT SP SZ YU

asper ganglbaueri Reitter, 1894b: 44 E: BH CR YU

NOTE:

For the distinguishing characters and distribution see Mikšić [1971], Mikšić \& Korpič [1985].

126. PAGE 296-297

PRINTED:

euphorbiae Germar, 1813: 131 (Saperda) E: AR AU BU CT

CZ HU IT MD RO SK ST UK A: KZ WS

histrionis Pic, 1917a: 11

imitans G. Müller, 1948: 76

intermedia Breuning, 1947c: 59 [HN] 
intermissa Breuning, 1962f: 212 [RN] moravica Kratochvíl, 1989: 1

MUST BE:

euphorbiae Germar, 1813: 131 (Saperda) E: AR AU BU CT

CZ HU IT MD RO SK ST UK A: KZ WS

imitans G. Müller, 1948: 76

intermedia Breuning, 1947c: 59 [HN]

intermissa Breuning, 1962f: 212 [RN]

and

histrionis Pic, 1917a: 11 E: AU CZ HU MD RO SK UK moravica Kratochvíl, 1989: 1 NOTE:

The incorporation of Oberea euphorbiaea histrionis Pic, 1917 into Oberea euphorbiaea is not acceptable, and was not argumented by Sama [2010a] — the reference to the position of $\mathrm{m}$. histrionis by Breuning [1962] was not enough.

\section{PAGE 299}

PRINTED:

nigriceps A. White, 1844: 425 (Saperda) A: HAI HKG ORR binhana Pic, 1923b: 12

sylvia Pascoe, 1858: 261

MUST BE:

nigriceps A. White, 1844: 425 (Saperda) A: HAI HKG ORR binhana Pic, 1923b: 12

nigromaculicollis Breuning, 1960b: 35 ("Chine: prov. Ngan-hwei")

sylvia Pascoe, 1858: 261

128. PAGE 299

PRINTED:

notata Pic, 1936a: 24 A: GUA JIA SCH ZHE

MUST BE:

notata Pic, 1936a: 24 A: GUA JIA SCH ZHE

kwangtungensis Breuning, 1960b: 37(“Chine: prov. Kwang-Tung Lien-ping")

rufoantennata Breuning, 1960b: 37(“Chine: prov. Cheking, Kiukiang")

129. PAGE 300

PRINTED:

walkeri Gahan, 1894d: 487 A: FUJ GUA GUI GUX HAI

HEN HKG JIX SCH YUN ZHE ORR

atroanalis Fairmaire, 1895: 189

bicoloritarsis Pic, 1923b: 11

changi Gressitt, 1942c: 5

robustior Pic, 1923b: 12

MUST BE:

walkeri Gahan, 1894d: 487 A: FUJ GUA GUI GUX HAI

HEN HKG JIX SCH SD YUN ZHE ORR

atroanalis Fairmaire, 1895: 189

atrosternalis Breuning, 1960b: 38 ("Chine: prov. Kwang-

Tung, Gao-Tung")

bicoloritarsis Pic, 1923b: 11

changi Gressitt, 1942c: 5

nigrobasicollis Breuning, 1960b: 38 ("Chine: prov.

Kwang-Tung, Lien Distr.")

robustior Pic, 1923b: 12

sikkimensis Breuning, 1960b: 38 ("Sikkim, Darjeeling")

130. PAGE 303

PRINTED:

circumdata circumdata Kraatz, 1882c: 337 A: AF KI KZ UZ TD

parterufipenis Breuning, 1967a: 2 (Pseudomallosia) sellata Ganglbauer, 1884: 567

circumdata pilosicollis Holzschuh, 1981: 107 A: KZ UZ
MUST BE

circumdata Kraatz, 1882c: 337 A: AF KI KZ UZ TD

parterufipenis Breuning, 1967a: 2 (Pseudomallosia)

sellata Ganglbauer, 1884: 567

pilosicollis Holzschuh, 1981: 107 A: KZ UZ

NOTE:

Ph. pilosicollis Holzschuh, 1981 was described as a subspecies of Ph. circumdata, but is in fact another species with totally different shape and punctation of prothorax, elytral punctation, body pubescence, shape of apical abdominal segments and many other characters.

\section{PAGE 303}

PRINTED:

armeniaca armeniaca Frivaldszky, 1878b: 10 [=1878a: 318 ] E: AB AR GG A: IS SY TR

armeniaca testaceovittata Pic, 1934c: 18 (Musaria) E: AB A: IN iranica Villiers, 1960b: 99

natali Lobanov, 1994a: 105

MUST BE (SEE NOTE 10 TO THE PAGE 51):

armeniaca Frivaldszky, 1878b: 10 [=1878a: 318$]$ E: AB AR GG A: IS SY TR

and

testaceovittata testaceovittata Pic, 1934c: 18 (Musaria) A: IN iranica Villiers, 1960b: 99

testaceovittata natali Lobanov, 1994a: 105 E: AB

\section{PAGE 304}

PRINTED:

pretiosa Faldermann, 1837: 298 E: AB AR GG A: IN IQ SY TR ninives Sama, 1994b: 33

nigroapicalis Breuning, 1944: 16

MUST BE:

pretiosa nigroapicalis Breuning, 1944: 16 A: IQ

ninives Sama, 1994b: 33

pretiosa pretiosa Faldermann, 1837: 298 E: AB AR GG A: IN SY TR NOTE:

For the characters and area of Phytoecia (Helladia) nigroapicalis Breuning, 1944 see Sama [1994b].

133. PAGE 306

PRINTED:

annulifera Pic, 1900q: 67

MUST BE (SEE NOTE 105 TO THE PAGES 823 AND 836):

annulifera T.Pic, 1900ㅁ: 67

134. PAGE 307

PRINTED:

icterica Schaller, 1783: 292 (Cerambyx) E: AU BH BU CR CZ FR GE HU IT MC MD PL PT RO SK SL SP ST SZ TR UK YU A: KZ WS ephippium Fabricius, 1792b: 317 (Saperda) ragusana Küster, 1844: 55 (Oberea) [DA]

MUST BE (SEE NOTE 13 TO THE PAGE 56):

icterica Schaller, 1783: 292 (Cerambyx) E: AU BH BU CR CZ FR GE HU IT MC MD PL PT RO SK SL SP ST SZ TR UK YU A: KZ WS

ephippium Fabricius, 1792b: 317 (Saperda) ragusana Küster, 1844: 55 (Oberea) [DA] subannulipes Pic, 1915: 11

135. PAGE 308

PRINTED:

annulata annulata Hampe, 1852 b: 315 (Phytoecia) E: AB A: IN TR 
angorensis Pic, 1952a: 2

wawerkana Reitter, 1905b: 239

MUST BE

annulata annulata Hampe, 1852b: 315 (Phytoecia) E: AB A

IN TR

annulata wawerkana Reitter, 1905b: 239 A: TR

angorensis Pic, 1952a: 2

NOTE: [2010].

See Rejzek \& Hoskovec [1999], Özdikmen \& Turgut

136. PAGE 329-330

PRINTED:

genus Saperda Fabricius, 1775: 184 type species Cerambyx carcharias Linnaeus, 1758

Amilia Mulsant, 1862: 376 type species Saperda phoca Frölich, 1793 (= Saperda similis Laicharting, 1784)

Anaerea Mulsant, 1839: 184 type species Cerambyx carcharias Linnaeus, 1758

Argalia Mulsant, 1862: 381 [HN] type species Saperda tremula Fabricius, 1775 (= Leptura octopunctata Scopoli, 1772)

Compsidia Mulsant, 1839: 182 type species Cerambyx populneus Linnaeus, 1758

Lopezcolonia Alonso-Zarazaga, 1998: 131 [RN] type species Saperda tremula Fabricius, 1775 (= Leptura octopunctata Scopoli, 1772)

alberti Plavilstshikov, 1915b: 80 [RN] A: ES FE GUA HEB JA JIL KZ MG NC SC TAI WS

decempunctata Gebler, 1830: 186 [HN]

balsamifera Motschulsky, 1860b: 151 (Compsidia) A: ES FE JA MG NC QIN NMO SC XIN XIZ innotatipennis Pic, 1910a: 2

bacillicornis Pesarini \& Sabbadini, 1997: 116 A: GAN QIN bilineatocollis Pic, 1924a: 19 A: FE GAN HEB HEN HUB JIA QIN SCH SHA SHG

carcharias Linnaeus, 1758: 394 (Cerambyx) E: AB AL AR AU BE BH BU BY CR CT CZ DE EN FI FR GB GE GG GR HU IR IT LA LT LU MC MD NL NR NT PL RO SK SL SP ST SV SZ TR UK YU A: ES FE GAN GUI HEI HUB HUN JIA JIL KZ MG NC SCH SHA TR WS XIN grisescens Mulsant, 1839: 184 villosa Gmelin, 1790: 1837 (Cerambyx)

jansonis Z. Wang, 2003: 382, 397 A: JIL

interrupta Gebler, 1825: 52 A: ES FE FUJ HEN JA JIL NC SC WS

laterimaculata Motschulsky, 1860b: 151

internescalaris Pic, 1934g: 36 A: SCH

kojimai Makihara \& Nakamura, 1985: 18 A: TAI

maculosa Ménétriés, 1832: 226 E: AB A: IN

nigra Gressitt, 1951a: 552 A: SHA

octomaculata Blessig, 1873: 221 A: ES FE JA MG SC SHN octopunctata Scopoli, 1772: 101 (Leptura) E: AB AL AR AU BE BH BU BY CR CT CZ FR GE GG GR HU IT MD PL RO SK SL SP ST SZ UK YU magnini Dayrem, 1928: 77

tiliae Schrank, 1798: 667

tremula Fabricius, 1775: 186

ohbayashii Podan1, 1963c: 62 [RN] A: JA

breuningi K. Ohbayashi, 1957: 14 [HN]

pallidipennis Gressitt, 1951a: 553 A: SHA

perforata Pallas, 1773: 723 (Cerambyx) E: AB AL AR AU BH BU BY CR CT CZ EN FI FR GE GG GR HU IT LA LT MD NR NT PL RO SK SP ST SV SZ TR UK N: AG A: ES FE IN KZ MG NE TR WS XIN albella Reitter, 1913d: 665 algerica Pic, 1903a: 8

decempunctata Goeze, 1777: 506 (Leptura)

duodecimpunctata Brahm, 1790: 176 (Leptura)

mesmini Pic, 1910c: 13

pallidipes Pic, 1904b: 9

rudolphi Cederhjelm, 1798: 92

seydlii Frölich, 1793: 135

populnea Linnaeus, 1758: 394 (Cerambyx) E: AB AL AR AU BE BH BU BY CT CZ DE EN FI FR GB GE GG GR HU IR IT LA LT LU MC MD NL NR NT PL PT RO SK SL SP ST SV SZ UK YU A: ANH ES FE FUJ GAN GUA HEB HEI HEN HUB IN JIA JIL KZ LIA MG NIN NMO SC SHA SHN SHX TR WS XIN NAR

betulina Geoffroy, 1785: 78

decempunctata DeGeer, 1775: 78 (Cerambyx)

populi Duméril, 1860: 607

salicis Zetterstedt, 1818: 258

punctata Linnaeus, 1767: 1067 (Cerambyx) E: AB AL AN

AR AU BH BU BY CR CT CZ EN FR GE GG GR HU IT

LA LT MA MC MD NT PL RO SK SL SP ST SZ TR UK

YU N: AG A: CY TR

gallica Pic, 1918d: 5

quercus ocellata Abeille de Perrin, 1895a: ccxxix A: IS JO SY TR

quercus quercus Charpentier, 1825: 224 E: BH BU GR YU scalaris hieroglyphica Pallas, 1773: 723 (Cerambyx) E: CT NT ST A: ES FE HEI JIL KZ LIA MG NC SHN WS XIN varia Gmelin, 1790: 1875 (Leptura) variegata Goeze, 1777: 506 (Leptura)

scalaris scalaris Linnaeus, 1758: 394 (Cerambyx) E: AB AL AR AU BE BH BU BY CR CT CZ DE EN FI FR GB GE GG GR HU IR IT LA LT LU MD NE NL NR NT PL RO SK SP ST SV SZ TR UK YU N: AG A: KZ TR algeriensis Breuning, 1952: 176 estellae Mulsant, 1839: 188

fenestrata Reineck, 1919: 72 xantha Demelt, 1960: 182

similis Laicharting, 1784: 31 E: AL AU BE BH BU BY CR CT CZ EN FI FR GE HU IT MC NR NT PL RO SK SL SP ST SV SZ UK YU A: ES FE KI KZ MG TD UZ WS albopubescens Pic, 1925d: 11

phoca Frölich, G. F. 1793: 139

simulans Gahan, 1888b: 64 A: HUN JIA JIL SCH

subobliterata Pic, 1910c: 13 A: FE HEI JA JIL SC mandschukuoensis Breuning, 1943b: 104

harbinensis Chou, Chao \& Chiang, 1983: 66 [RN]

subscalaris Breuning, 1952: 179 A: YUN

tetrastigma Bates, 1879b: 466 A: JA SC TAI yezoana Matshushita, 1933: 402 (Cagosima) viridipennis Gressitt, 1951a: 554 A: SHA MUST BE:

genus Saperda Fabricius, 1775: 184 type species Cerambyx carcharias Linnaeus, 1758

subgenus Compsidia Mulsant, 1839: 182 type species Cerambyx populneus Linnaeus, 1758

balsamifera Motschulsky, 1860b: 151 (Compsidia) A: ES FE JA MG NC QIN NMO SC XIN XIZ innotatipennis Pic, 1910a: 2

bacillicornis Pesarini \& Sabbadini, 1997: 116 A: GAN QIN bilineatocollis Pic, 1924a: 19 A: FE GAN HEB HEN HUB JIA QIN SCH SHA SHG

nigra Gressitt, 1951a: 552 A: SHA

populnea Linnaeus, 1758: 394 (Cerambyx) E: AB AL AR AU BE BH BU BY CT CZ DE EN FI FR GB GE GG GR HU IR IT LA LT LU MC MD NL NR NT PL PT RO SK SL SP ST SV SZ UK YU A: ANH ES FE FUJ GAN GUA 
HEB HEI HEN HUB IN JIA JIL KZ LIA MG NIN NMO SC SHA SHN SHX TR WS XIN NAR betulina Geoffroy, 1785: 78

decempunctata DeGeer, 1775: 78 (Cerambyx) populi Duméril, 1860: 607

salicis Zetterstedt, 1818: 258

quercus ocellata Abeille de Perrin, 1895a: ccxxix A: IS JO SY TR

quercus quercus Charpentier, 1825: 224 E: BH BU GR YU

subgenus Lopezcolonia Alonso-Zarazaga, 1998: 131 [RN] type species Saperda tremula Fabricius, 1775 (= Leptura octopunctata Scopoli, 1772)

Argalia Mulsant, 1862: 381 [HN] type species Saperda tremula Fabricius, 1775 (= Leptura octopunctata Scopoli, 1772)

alberti Plavilstshikov, 1915b: 80 [RN] A: ES FE GUA HEB

JA JIL KZ MG NC SC TAI WS

decempunctata Gebler, 1830: $186[\mathrm{HN}]$

internescalaris Pic, 1934g: 36 A: SCH

interrupta Gebler, 1825: 52 A: ES FE FUJ HEN JA JIL NC SC WS

laterimaculata Motschulsky, 1860b: 151

kojimai Makihara \& Nakamura, 1985: 18 A: TAI

maculosa Ménétriés, 1832: 226 E: AB A: IN

octomaculata Blessig, 1873: 221 A: ES FE JA MG SC SHN

octopunctata Scopoli, 1772: 101 (Leptura) E: AB AL AR

AU BE BH BU BY CR CT CZ FR GE GG GR HU IT MD

PL RO SK SL SP ST SZ UK YU

magnini Dayrem, 1928: 77

tiliae Schrank, 1798: 667

tremula Fabricius, 1775: 186

ohbayashii Podan1, 1963c: 62 [RN] A: JA

breuningi K. Ohbayashi, 1957: 14 [HN]

pallidipennis Gressitt, 1951a: 553 A: SHA

perforata Pallas, 1773: 723 (Cerambyx) E: AB AL AR AU BH BU BY CR CT CZ EN FI FR GE GG GR HU IT LA LT MD NR NT PL RO SK SP ST SV SZ TR UK N: AG A: ES FE IN KZ MG NE TR WS XIN

albella Reitter, 1913d: 665

algerica Pic, 1903a: 8

decempunctata Goeze, 1777: 506 (Leptura)

duodecimpunctata Brahm, 1790: 176 (Leptura)

mesmini Pic, 1910c: 13

pallidipes Pic, 1904b: 9

rudolphi Cederhjelm, 1798: 92

seydlii Frölich, 1793: 135

punctata Linnaeus, 1767: 1067 (Cerambyx) E: AB AL AN AR AU BH BU BY CR CT CZ EN FR GE GG GR HU IT LA LT MA MC MD NT PL RO SK SL SP ST SZ TR UK YU N: AG A: CY TR gallica Pic, 1918d: 5

scalaris hieroglyphica Pallas, 1773: 723 (Cerambyx) E: CT NT ST A: ES FE HEI JIL KZ LIA MG NC SHN WS XIN varia Gmelin, 1790: 1875 (Leptura) variegata Goeze, 1777: 506 (Leptura)

scalaris scalaris Linnaeus, 1758: 394 (Cerambyx) E: AB AL AR AU BE BH BU BY CR CT CZ DE EN FI FR GB GE GG GR HU IR IT LA LT LU MD NE NL NR NT PL RO SK SP ST SV SZ TR UK YU N: AG A: KZ TR algeriensis Breuning, 1952: 176 estellae Mulsant, 1839: 188 fenestrata Reineck, 1919: 72 xantha Demelt, 1960: 182

simulans Gahan, 1888b: 64 A: HUN JIA JIL SCH subobliterata Pic, 1910c: 13 A: FE HEI JA JIL SC mandschukuoensis Breuning, 1943b: 104 harbinensis Chou, Chao \& Chiang, 1983: 66 [RN]

subscalaris Breuning, 1952: 179 A: YUN

tetrastigma Bates, 1879b: 466 A: JA SC TAI

yezoana Matshushita, 1933: 402 (Cagosima)

viridipennis Gressitt, 1951a: 554 A: SHA

subgenus Saperda Fabricius, 1775: 184 type species Cerambyx carcharias Linnaeus, 1758

Amilia Mulsant, 1862: 376 type species Saperda phoca Frölich, 1793 (= Saperda similis Laicharting, 1784)

Anaerea Mulsant, 1839: 184 type species Cerambyx carcharias Linnaeus, 1758

carcharias Linnaeus, 1758: 394 (Cerambyx) E: AB AL AR AU BE BH BU BY CR CT CZ DE EN FI FR GB GE GG GR HU IR IT LA LT LU MC MD NL NR NT PL RO SK SL SP ST SV SZ TR UK YU A: ES FE GAN GUI HEI HUB HUN JIA JIL KZ MG NC SCH SHA TR WS XIN grisescens Mulsant, 1839: 184 villosa Gmelin, 1790: 1837 (Cerambyx)

jansonis Z. Wang, 2003: 382, 397 A: JIL

similis Laicharting, 1784: 31 E: AL AU BE BH BU BY CR CT CZ EN FI FR GE HU IT MC NR NT PL RO SK SL SP ST SV SZ UK YU A: ES FE KI KZ MG TD UZ WS albopubescens Pic, 1925d: 11

phoca Frölich, G. F. 1793: 139

\section{PAGE 328}

PRINTED:

sulphurata Gebler, 1825: 52 (Saperda) E: CT A: ES FE HEB HEN HUB JA JIL KZ MG NC SC SCH SHA SHN SHX TAI WS

galathea J. Thomson, 1865a: 566 (Glenea)

nigrocincta Pic, 1915e: 10

semivittata Pic, $1915 \mathrm{e}: 10$

vitiphaga Holzschuh, 2003: 237 A: SHA

yuasai Gressitt, 1935b: 176 (Praolia) A: JA

MUST BE:

sulphurata Gebler, 1825: 52 (Saperda) E: CT A: ES FE HEB HEN HUB JA JIL KZ MG NC SC SCH SHA SHN SHX TAI WS

galathea J. Thomson, 1865a: 566 (Glenea)

nigrocincta Pic, $1915 \mathrm{e}: 10$

semivittata Pic, 1915e: 10

vuasai Gressitt, 1935b: 176 (Praolia)

vitiphaga Holzschuh, 2003: 237 A: SHA

NOTE:

According to N. Ohbayashi [personal message, 2010] Menesia sulphurata (Gebler, 1825) = Praolia yuasai Gressitt, 1935b. The synonyms were published [Hayashi, 1974b].

138. PAGE 329-333

PRINTED:

gilvipes Faldermann, 1837: 290 (Anaetia) E: AB AR GG ST UK A: IN TM

and

praeustus praeustus Linnaeus, 1758: 399 (Leptura) E: AB AL AR AU BE BH BU BY CR CT CZ DE EN FI FR GB GE GG GR HU IR IT LA LS LT LU MC MD NL NR NT PL PT RO SK SL SL SP ST SV SZ TR UK YU A: ES KZ MG SY TR WS

anatolicus Özdikmen \& Turgut, 2008e: 627

angorensis Pic, $1918 \mathrm{~d}: 11$

inapicalis Pic, 1891b: 37

mesmini Pic, 1928c: 6

muehlfeldi Mulsant, 1862: 348 (Polyopsia)

niger Kraatz, 1859: 57

pilosus Geoffroy, 1785: 78 (Leptura) 
ustulatus Hagenbach, 1822: 11 (Saperda) vicinus Pic, 1928c: 6

MUST BE:

gilvipes gilvipes Faldermann, 1837: 290 (Anaetia) E: AB AR GG ST UK A: IN TM

gilvipes niger Kraatz, 1859: 57 E: IT FR

muehlfeldi Mulsant, 1862: 348 (Polyopsia) and

praeustus praeustus Linnaeus, 1758: 399 (Leptura) E: AB AL AR AU BE BH BU BY CR CT CZ DE EN FI FR GB GE GG GR HU IR IT LA LS LT LU MC MD NL NR NT PL PT RO SK SL SL SP ST SV SZ TR UK YU A: ES KZ MG SY TR WS

anatolicus Özdikmen \& Turgut, 2008e: 627

angorensis Pic, 1918d: 11

inapicalis Pic, 1891b: 37

mesmini Pic, $1928 \mathrm{c}: 6$

pilosus Geoffroy, 1785: 78 (Leptura)

ustulatus Hagenbach, 1822: 11 (Saperda)

vicinus Pic, 1928c: 6

\section{PAGE 730}

One publication is referred as two different in different years.

PRINTED:

Hammarström E. R. 1892: Bidrag till kännedom of sydvestra Sibiriens insektfauna. Förtecking öfver i Minusinska kretsen och angränsande delar af Mongoliet af K. J. Ehrenberg och R. E. Hammarström sommaren 1885 insamlade Cerambycider. Öfversigt af Finska Vetenskaps-Societetensd Förhandlingar 34 [1891-1892]: 185-195.

Hammarström R. 1893: Bidrag till kännedom af sydvestra Sibiriens insektfauna. Öfversigt af Finska Vetenskap Societetens Förhandlingar 34: 185-195. [1893].

No taxons in the catalogue are referred to Hammarström

140.

MISSING REFERENCE:

Hayashi M. 1982: The Cerambycidae of Japan (Col.)

(13). The Entomological Review of Japan 37(2): 141-152. for the names: 130)

limbaticollis stephani Hayashi, 1982: 152 [RN] A: JA (p.

makiharai Hayashi, 1982: 151 (Euchlanis) [RN] A: TAI

(p. 205)

daurica sakaii Hayashi, 1982: 149 A: JA (p. 215)

yagii Hayashi, 1982: 147 A: JA (p. 216)

141. PAGE 730

PRINTED:

Krynicki J. 1832: Enumeratio Coleopterorum Rossiae meridionalis et praecipue in Universitatis Caesareae Charkoviensis circulo obvenientium, quae annorum 1827-1831 spatio observavit. Bulletin de la Société Impériale des Naturalistes de Moscou 5: 65-179, pls II-III.

Krynicki J. 1834: Enumeratio Coleopterorum Rossiae meridionalis et praecipue in Universitatis Caesareae Charkoviensis circulo obvenientium, quae annorum 1827-1831 spatio observavit. Bulletin de la Société Impériale des Naturalistes de Moscou 7: 166-173.

MUST BE:

Krynicki J. [I.], 1832: Enumeratio Coleopterorum Rossiae meridionalis et praecipue in Universitatis Caesareae Charkoviensis circulo obvenientium, quae annorum 1827 1831 spatio observavit. - Bulletin de la Société Impériale des Naturalistes de Moscou, 5: [+3pages] 68-179, pls II-III.

[Krynicki J. I.] 1834 [no author's name in the publication]: Addenda et nonnula synonyma Rossiae meridionalias Coleopterorum. (vide Bulletin. Vol. v. p. 69). - Bulletin de la Société Impériale des Naturalistes de Moscou, 7: 166-173.

\section{PAGE 734}

PRINTED:

Hayashi M. \& Villiers A. 1985b: Revision of the Asian Lepturinae (Coleoptera: Cerambycidae) With special reference to the type specimens' inspection. Part II. Bulletin of Osaka Jonan Women's Junior College 22: 1-20.

MUST BE:

Hayashi M. \& Villiers A. 1987: Revision of the Asian Lepturinae (Coleoptera: Cerambycidae) With special reference to the type specimens' inspection. Part II. Bulletin of Osaka Jonan Women's Junior College 22: 1-20.

\section{PAGE 771}

PRINTED:

Lameere A. 1912a: Révision des prionides. Vingt-etunième mémoire: Anacolines. Vingt-deuxième mémoire. Addenda et corrigenda. Mémoires de la Société Entomologique de Belgique 12: 1-188.

MUST BE:

Lameere A. 1912a: Révision des prionides. Vingt-etunième mémoire: Anacolines. Vingt-deuxième mémoire. Addenda et corrigenda. Mémoires de la Société Entomologique de Belgique 21: 1-188.

\section{PAGE 772}

PRINTED:

Lazarev M.A. 2009: Armenian Dorcadion (Coleoptera: Cerambycidae). Studies and Reports of District Museum Prague-East Taxonomic Series 5: 197-220.

MUST BE:

Lazarev M.A. 2008: Zametki po spornym voprosam sistematiki i rasprostranenia zhukov-usachey (Coleoptera, Cerambycidae) Rossii i sopredelnyh stran. Pp. 129-136. In: Aktualnye problemy prioritetnyh napravleniy razvitiya estestvennyh nauk. Sbornik statey. Moskva, Izdatelstvo «Prometey» MPGU: 220ñ.

Lazarev M.A. 2009: Armenian Dorcadion (Coleoptera: Cerambycidae) of "cinerarium-group". Studies and Reports of District Museum Prague-East Taxonomic Series 5: 197-220.

\section{PAGE 823}

PRINTED:

Pic M. 1898v: Description d'une variété nouvelle de Phytoecia (Col.). Bulletin de la Société Entomologique de France 1898: 334-335.

MUST BE:

Pic M. 1898v: Diagnose d'une variété nouvelle de Phytoecia (Col.). Bulletin de la Société Entomologique de France 1898: $334-335$.

\section{PAGE 823 AND 836}

One publication is referred as two different with different authors:

PRINTED:

Pic M. 1900q: Diagnosen verschiedener Phytoecia aus dem Orient. Entomologische Nachrichten 26: 67-68.

and

Pic T. 1900b: Diagnosen verschiedener Phytoecia aus dem Orient. Entomologische Nachrichten 26: 67-68.

The second case is correct. 


\section{PAGE 833}

PRINTED:

Pic M. 1932g: Lepturinen-Studien (Col., Cerambycidae). I. Časopis Československé Společnosti Entomologické 29: 87-88.

Such publication does not exist, and no name in the catalog is dated as «Pic, 1932g»

It is modified name of Plavilstshikov's publication, which is absent in the Catalog. The exact reference is:

Plavilstshikov N.N. 1932: Lepturinen-Studien (Col., Cerambycidae). I. Časopis Československé Společnosti Entomologické 29: 87-88, 174-175.

\section{PAGE 833} years:

One publication is referred as two different in different PRINTED:

Pic M. 1933i: Sur Evodinus interrogationis L. (1). Pp. 2132. Matériaux pour servir à l'étude des longicornes. 1lème cahier. Saint-Amand (Cher): Imprimerie Bussière, 16 pp. and

Pic M. 1934f: Sur Evodinus interrogationis L. (I). Pp. 21-32. Matériaux pour servir à l'étude des longicornes. 11 ème cahier, 2 me partie. Saint-Amand (Cher): Imprimerie Bussière, 17-32 pp.

The correct is the second one! So, all references to Pic, 1933i are connected with Pic, $1934 f$

\section{PAGE 837}

PRINTED:

Plavilstshikov N.N. 1932a: Cerambycidae II. Cerambycinae: Cerambycini II. Bestimmungs-Tabellen der europäischen Coleopteren. Heft 102. Troppau: Edmund Reitter's Nachfolger Emmerich Reitter, 145 pp.

No taxons from that publication are in the Catalog! MUST BE:

Plavilstshikov N.N. 1932a: Lepturinen-Studien (Col., Cerambycidae). I. Časopis Československé Společnosti Entomologické 29: 87-88, 174-175.

\section{PAGE 875}

PRINTED:

Tournier H. 1872: Catalogue des longicornes récoltés par M. Théophile Deyrolle, en Imerétie, Mingrélie et Georgie, et description des espèces nouvelles. Revue et Magasin de Zoologie (2) 23: 257-261, 276-292, 338-349. MUST BE:

Tournier H. 1872: Catalogue des longicornes récoltés par M. Théophile Deyrolle, en Imerétie, Mingrélie et Georgie, et description des espèces nouvelles. Revue et Magasin de Zoologie Pure et Appliquée (2) 23: 257-261, 276-292, 338-349.

ACKNOWLEDGEMENTS. I am very grateful to Alexey Gusakov and Andrey Ozerov (Zoological Museum of Moscow University), Andrery Lobanov (Zoological Institute, Sankt-Petersnurg) for providing me with the opportunity to study museums' materials. My special thanks to Alain Drumont, Ivan Löbl, Nobuo Ohbayashi, Ales Smetana and Gianfranco Sama for valuable friendly consultations on many taxonomy problems.

\section{References}

Alekseev V.L. 2007. Longhorn beetles (Coleoptera, Cerambycidae) of Kaliningrad region // Acta Biol. Univ. Daugavp. Vol.7. No.1. P.37-62.
Alexandrovich O.R., Lopatin I.K., Pisanenko A.D., Tzinkevich V.A., Snitko S.M. 1996. Katalog zhestkokrylykh (Coleoptera, Insecta) Belarusi. Minsk. FFI RB. 103 p. [in Russian].

Danilevsky M. L. 1984. Opisanie lichinki Prionus komarovi Dohrn. (Coleoptera, Cerambycidae) larvae // Biologicheskie nauki. No.12. P.33-35 [in Russian].

Danilevsky M.L. 2009a. Taksony zhukov-usachey (Coleoptera, Cerambycidae) vidovoy gruppy, opisannye N.N. Plavilstshikovym, i ikh tipy v kollektziyakh Zoologicheskogo Muzeya Moskovskogo gosudarstvennogo universiteta i Zoologicheskogo instituta RAN v Sankt-Peterburge // Entomologicheskoe Obozrenie. Vol.88. Is.3. P.630-663 [in Russian].

Danilevsky M.L. 2009b. Species Group Taxa of Longhorned Beetles (Coleoptera,Cerambycidae) Described by N. N. Plavilstshikov and Their Types Preserved in the Zoological Museum of the Moscow State University and in the Zoological Institute of the Russian Academy of Sciences, St. Petersburg // Entomological Review. Vol.89. No.6. P.689-720.

Drumont A., Grifnee V. 2005. Une nouvelle espèce de Longicornes pour la faune de Belgique: Gaurotes (Carilia) virginea (Linnaeus, 1758) (Coleoptera, Cerambycidae) // Lambillionea. Vol.105. No.3. T.2. P.433-436.

Donisthorpe H. 1905. Gramoptera holomelina, Pool, a good species // Entomologist's Record and Journal of Variation. Vol.17. P. $182-183$

Hayashi M., Villiers A. 1987. Revision of the Asian Lepturinae (Coleoptera: Cerambycidae) With special reference to the type specimens' inspection. Part II // Bulletin of Osaka Jonan Women's Junior College. Vol.22. P.1-20. Pls.1-3.

Kraatz G. 1876 [Fußnote, S.318] // L.Heyden. 1876. Die Cortodera- (Muls.) und Grammoptera- (Serv.) Arten // Deutsche Entomologische Zeitschrift. Bd.20. S.317-320.

Lazarev M.A. 2008. Zametki po spornym voprosam sistematiki i rasprostranenia zhukov-usachey (Coleoptera, Cerambycidae) Rossii i sopredelnyh stran // Aktualnye problemy prioritetnyh napravleniy razvitiya estestvennyh nauk. Sbornik statey. Moskva: Izdatelstvo «Prometey» MPGU. P.129-136 [in Russian].

Löbl I., Smetana A. (ed.) 2010. Catalogue of Palaearctic Coleoptera. Vol.6. Chrysomeloidea. Stenstrup. Apollo Books. 924pp.

Makihara H. 2007. Dorcaschematini // N. Ohbayashi \& T Niisato (eds.). Longicorn beetles of Japan. Tokai Univ. Press, Kanagawa. P.310-311.

Mamaev B.M., Danilevsky M.L. 1975. Lichinki zhukov-drovosekov. Moscow: Nauka. 282 p. [in Russian].

Mikšiæ R. 1971. Katalog der Bockkäfer (Cerambycidae) Jugoslawiens (Insecta-Coleoptera). Sarajevo. $70 \mathrm{~S}$.

Özdikmen H. 2003. The Genus Cortodera Mulsant, 1863 (Cerambycidae: Coleoptera) in Turkey // Phytoparasitica. Vol.31. No.5. P.433-441.

Özdikmen H., Turgut S. 2010. An overview on the palaearctic subgenus Phytoecia (Pilemia) Fairmaire, 1864 with a new species Phytoecia (Pilemia) samii sp.n. from Turkey (Coleoptera: Cerambycidae: Lamiinae) // Munis Entomology \& Zoology. Vol.5. No.1. P.90-108.

Plavilstshikov N.N. 1932. Zhuki-drovoseki vrediteli drevesiny. Moskva-Leningrad: Gosudarstevennoe Lesnoe Tekhnicheskoe Izdatel'stvo. 200 p. [in Russian].

Pool C. J. C. 1905. Grammoptera ruficornis ab. holomelina, n. ab., a wholly black form of Grammoptera, Ser., not hitherto recorded // Entomologist's Record and Journal of Variation. Vol.17. P.133.

Rejzek M., Hoskovec M. 1999. Cerambycidae of Nemrut Dağı National Park (Anatolia, South-East Turkey) // Biocosme Mésogéen, Nice. Vol.15. No.4. P.257-272.

Rost C. 1892. Brachyta bifasciata Ol. var. caucasica Rost // Entomologische Nachrichten. Bd.18. Nr.6. S.81.

Sama G., Buse J., Orbach E., Friedman A.L.L., Rittner O., Chikatunov V. 2010. A new catalogue of the Cerambycidae (Coleoptera) of Israel with notes on their distribution and host plants // Munis Entomology \& Zoology. Vol.5. No.1. P. 1-55. 
Santos-Silva A., Hovore F.T. 2007. Divisão do gênero Distenia Lepeletier \& Audinet-Serville, notas sobre a venação alar em Disteniini, homonímias, sinonímia e redescrições (Coleoptera, Cerambycidae, Disteniinae) // Papéis Avulsos de Zoologia. Vol.47. No.1. P.1-29.

Stierlin W. G. 1898. Fauna coleopterorum helvetica. Die KäferFauna der Schweiz nach der analytischen Methode. 2 Teil. Schaffhausen. Bolli and Bocherer. S.xii+662.

Švácha P. 1987. In: Švácha P. \& Danilevsky M.L. 1987. Ceramby- coid larvae of Europe and Soviet Union (Coleoptera, Cerambycoidea). Part I // Acta Universitatis Carolinae. Vol.30 [1986]. No.1-2. P.1-177.

Tsherepanov A. I. 1996. 104. Sem. Cerambycidae - Usachi ili drovoseki // P.A. Ler (ed.). Opredelitel nasekomykh Dalnego Vostoka Rossii. Vol.3. Zhestkokrylye, ili zhuki. Chast 3. Vladivostok: Dal'nauka. P.56-140. (the text was arranged by G.O. Krivolutzkaya and A.L. Lobanov on the base of a manuscript by Tsherepanov) [in Russian]. 